\title{
SINGULAR PULSE WAVE BIFURCATIONS FROM FRONT AND BACK WAVES IN BISTABLE REACTION-DIFFUSION SYSTEMS
}

\author{
Hideo Ikeda
}

\begin{abstract}
Singular perturbation methods are applied to show a bifurcation of traveling pulse waves from front and back waves with the same velocity. In our proof, the behavior of the stable and unstable manifolds with respect to the equilibrium points as they cross are given, and these play an essential part in proving the existence and stability properties of traveling pulse waves.
\end{abstract}

\section{Introduction}

In the framework of nonequilibrium systems, reaction-diffusion systems have been proposed to model spatial and temporal pattern dynamics in the fields of chemical reactions, neurophysiology, morphogenesis, combustion, solidification, etc. It is recognized that under the interaction of two simple mechanisms, reaction and diffusion, very complex and very beautiful spatial and temporal patterns appear.

This paper studies traveling pulse solutions for a system of reaction-diffusion equations of the form

$$
\left.\begin{array}{rl}
\varepsilon u_{t} & =\varepsilon^{2} u_{x x}+f(u, v) \\
v_{t} & =D v_{x x}+g(u, v)
\end{array}\right\} \quad(t, x) \in \mathbf{R}_{+} \times \mathbf{R} .
$$

This system contains two physical parameters $\varepsilon$ and $D$. We assume that $\varepsilon$ is a sufficiently small positive parameter and $D$ is a positive parameter. The reaction terms $f$ and $g$ are taken to be typical of excitable media (see Figure 1). Figure 1(a) shows the mono-stable case, and Figure 1(b) does the bi-stable case, depending on the number of stable constant stationary solutions of (1.1). For this system, an important problem is to study the existence and the stability properties of traveling wave solutions, which gives us an understanding of high-dimensional wave propagation such as spiral patterns and labyrinthine patterns.

First, let us consider the mono-stable case. When $D=0$, this system is called the FitzHugh-Nagumo equations, and the existence of stable traveling pulse solutions is well-known. But when $D \neq 0$, it is difficult to show the existence and the stability property of traveling pulse solutions rigorously. When $D=O(\varepsilon)\left(=\varepsilon D_{1}\right)$, this system is known as the Belousov-Zhabotinskii reaction equations. It has been believed that there exist stable traveling pulse solutions for this system. In fact, Dockery and Keener [1] showed that traveling pulse solutions and standing pulse solutions coexist for the system with piecewise linear $f$ and $g$. Furthermore, they showed that the branch of traveling pulse solutions bifurcates from the one for standing pulse solutions, and no traveling pulse solutions exist for large $D_{1}$; they used a formal perturbation analysis and numerical methods. On the other hand, when $D=O(1)$, this system is

Received December 15, 1994, revised July 7, 1995.

1991 Mathematics Subject Classification: 35B25, 35B32, 35K57.

Key words and phrases: traveling pulses, singular limit, bifurcation, crossing behavior of stable and unstable manifolds, bistable reaction-diffusion systems. 


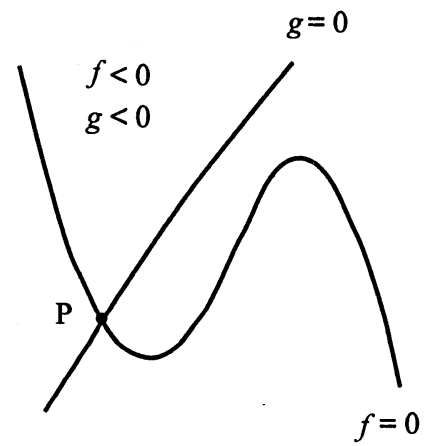

(a)

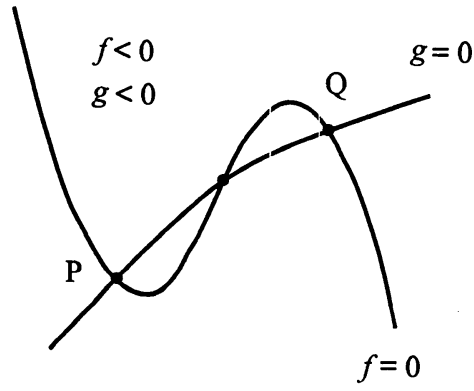

(b)

FIgURE 1. Typical functional forms of excitable media: (a) monostable, (b) bi-stable.

known as lateral inhibition, which implies the existence of standing pulse solutions. These results suggest that no traveling pulse solutions exist when $D=O(1)$.

In this paper, we treat the bi-stable case. It is to be expected that the results similar to the above hold with respect to each stable equilibrium point. Here we shall show stronger results than the mono-stable case. That is, when $D=O(1)$, there exist traveling pulse solutions under the special nonlinearities $f$ and $g$. Replacing $x$ by $x / \sqrt{D}$, we can rewrite (1.1) as the following $\varepsilon-\tau$ system:

$$
\left.\begin{array}{rl}
\varepsilon \tau u_{t} & =\varepsilon^{2} u_{x x}+f(u, v) \\
v_{t} & =v_{x x}+g(u, v)
\end{array}\right\} \quad(t, x) \in \mathbf{R}_{+} \times \mathbf{R}
$$

where $\varepsilon / \sqrt{D}$ and $\sqrt{D}$ are regarded as $\varepsilon$ and $\tau$, respectively. Introducing the traveling coordinate $z=x+c t$, we rewrite (1.2) with a parameter $c$ :

$$
\left.\begin{array}{rl}
\varepsilon^{2} u_{z z}-\varepsilon c \tau u_{z}+f(u, v) & =0 \\
v_{z z}-c v_{z}+g(u, v) & =0
\end{array}\right\} \quad z \in \mathbf{R} .
$$

We can deal with the three kinds of traveling wave solutions $(u, v)(z)$ of $(1.2)$ with velocity $c$ : (a) traveling front wave, (b) traveling back wave, and (c) traveling pulse wave. The first one means a solution $(u, v)(z)$ of $(1.3)$ satisfying $(u, v)(-\infty)=P$ and $(u, v)(\infty)=Q$, while the second one satisfies the opposite boundary conditions $(u, v)(-\infty)=Q$ and $(u, v)(\infty)=P$. The third one satisfies the boundary conditions $(u, v)( \pm \infty)=P$ or $Q$. Note that a traveling front wave with velocity $c$ becomes a traveling back with velocity $-c$ using a suitable transformation. We know already that there exist traveling front and back waves of (1.2) with the bi-stable nonlinearity (see [5]). If we take $f$ and $g$ to be

$$
\begin{aligned}
f(u, v) & =u(1-u)(u-a)-v(0<a<1 / 2), \\
g(u, v ; \gamma) & =u-\gamma v
\end{aligned}
$$

(see Figure 2), we can get the global branches of front and back waves, $c$ versus $\gamma$ (see Figure 6). Here $\gamma$ is a positive parameter satisfying $\gamma>\gamma_{0}$, and $\gamma_{0}, \gamma_{1}$, and $\gamma_{2}$ are the critical values as shown in Figure 3.

At $\gamma=\gamma_{1}$, front and back waves with the same velocities $c$ coexist because of the odd symmetry of $f$ and $g$. This situation suggests that traveling pulse-like waves satisfying $(u, v)( \pm \infty)=P$ or $Q$ exist whose length of excited regions are infinite. This also suggests the possibility of the existence of traveling pulse waves, whose length of 


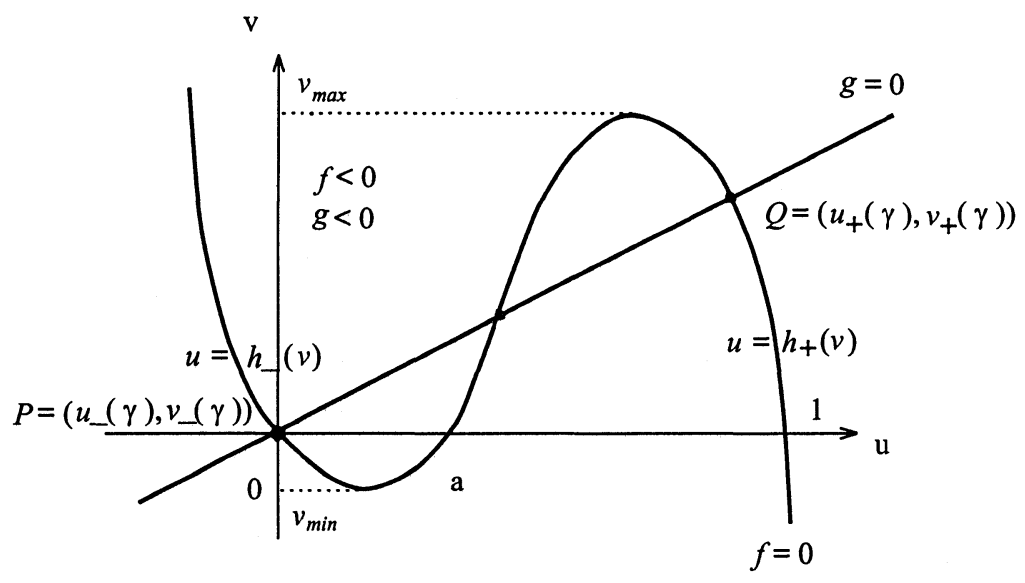

FIgURE 2. Functional forms of $f=0$ and $g=0$ in (1.3).

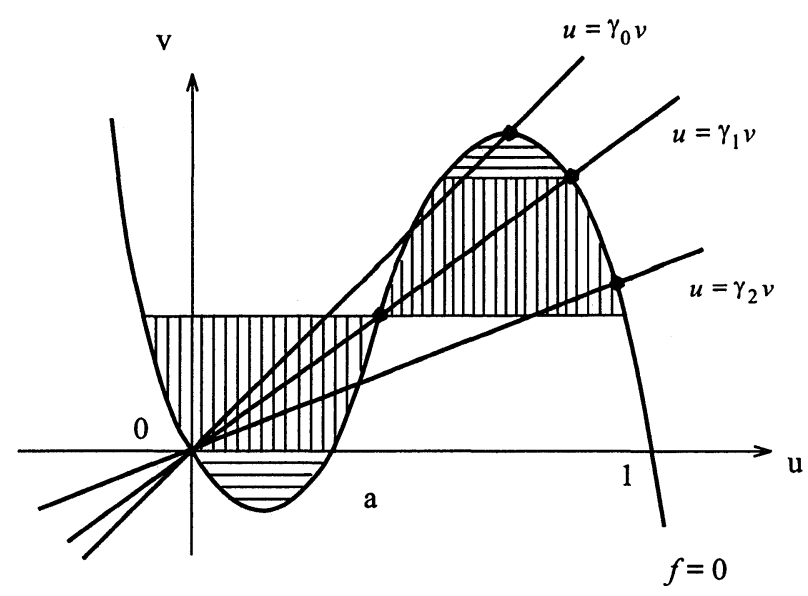

Figure 3. Critical values of $\gamma$.

excited regions are very long but finite, in a neighborhood of $\gamma_{1}$ (see Figure 7). This conjecture was proved affirmatively in [6] by using homoclinic bifurcation theory. The system (1.3) can be rewritten as an equivalent four-dimensional dynamical system

$$
\begin{aligned}
\frac{d}{d z} \mathbf{V} & =\mathbf{F}(\mathbf{V} ; \varepsilon ; \tau, c) \quad z \in \mathbf{R}, \\
\mathbf{V}( \pm \infty) & =\mathbf{P} \quad(\text { or } \mathbf{Q})
\end{aligned}
$$

for $\mathbf{V}=\left(u, \varepsilon u_{z}, v, v_{z}\right)$. Then a traveling pulse wave of (1.2) corresponds to a homoclinic orbit of $(1.5)$ based on $\mathbf{P}=(0,0,0,0)$ (or $\mathbf{Q}=\left(u_{+}(\gamma), 0, v_{+}(\gamma), 0\right)$ ). Kokubu et al. [6] showed the bifurcation of front and back waves by using distance-like functions, which are called separations, of the stable and unstable manifolds with respect to $\mathbf{P}$ and $\mathbf{Q}$. Next, by the aid of these separations, they showed the bifurcation of pulse waves at $\gamma=\gamma_{1}$. But the crossing behavior of the stable and unstable manifolds with respect to $\mathbf{P}$ or $\mathbf{Q}$ were not obtained there, and that plays an essential role for locally unique existence and the stability property. Here, we want to obtain this information by using an analytic singular perturbation method which is different from [6] (see §4). 
In this paper, we only consider the singular limit problem which has important information about the existence and the stability property of an exact solution. The existence and the stability properties of these solutions will be given in [4].

In $\S 2$, we give a short summary of the existence results for traveling front and back waves and will see that traveling front and back waves with the same velocity coexist at $\gamma=\gamma_{1}$. In $\S 3$, we consider the singular limit problem for traveling pulse waves and show that singular limit pulse waves bifurcate from singular limit front and back waves at $\gamma=\gamma_{1}$. In $\S 4$, the crossing behavior (see (4.25), (4.26)) of the stable and unstable manifolds of the equilibrium points for the singular limit problem are given and are good approximations of those for the full system (1.5). We give the most important information for proving the existence of an exact solution and its stability property. Finally, in $\S 5$, we make a few comments on our results.

For simplicity, we assume that the nonlinearities $f$ and $g$ are as in (1.4). But our assumptions will be weakened to more general nonlinearities such that singular limit traveling front and back waves with the same velocity coexist at some value of a physical parameter (see §3). In Figure 2, $u=h_{-}(v)$ and $u=h_{+}(v)$ denote the left and the right branches of $f=0$, respectively. Two stable constant states are $P=(0,0)$ and $Q=\left(u_{+}(\gamma), v_{+}(\gamma)\right)$. Hereafter, we write $(0,0)$ as $\left(u_{-}(\gamma), v_{-}(\gamma)\right)$ for a short notation. Define $\mathfrak{F}(v)=\int_{h_{-}(v)}^{h_{+}(v)} f(u, v) d u$ for $v \in\left(v_{\min }, v_{\max }\right)$. Then, it is easily seen that $\mathfrak{F}(v)$ has a unique isolated zero at $v^{*} \in\left(v_{\min }, v_{\max }\right)$.

We use the following function spaces. Let $\mathbf{I}=\mathbf{R}_{-}, \mathbf{R}_{+}$, or $\mathbf{R}, \varepsilon$ and $\rho$ be positive numbers, and $n$ be an integer. Let

$$
X_{\rho, \varepsilon}^{n}(\mathbf{I})=\left\{u \in C^{n}(\mathbf{I})\left|\|u\|_{X_{\rho, \varepsilon}^{n}(\mathbf{I})}=\sum_{k=0}^{n} \sup _{x \in \mathbf{I}} e^{\rho|x|}\right|\left(\varepsilon \frac{d}{d x}\right)^{k} u(x) \mid<\infty\right\} .
$$

\section{Front and back waves}

In this section, we give a short summary of the existence results for traveling front and back waves of our previous paper [5]. As stated above, the existence of a front wave with velocity $c$ implies the existence of a back wave with velocity $-c$. So we consider only a front wave for a while. Introducing the traveling coordinate $z=x+c t$, we find that a traveling front wave with velocity $c$ satisfies the following equations:

$$
\left.\begin{array}{rl}
\varepsilon^{2} u_{z z}-\varepsilon c \tau u_{z}+f(u, v) & =0 \\
v_{z z}-c v_{z}+g(u, v ; \gamma) & =0
\end{array}\right\} \quad z \in \mathbf{R}
$$

with boundary conditions

$$
(u, v)(-\infty)=P \equiv\left(u_{-}(\gamma), v_{-}(\gamma)\right), \quad(u, v)(\infty)=Q \equiv\left(u_{+}(\gamma), v_{+}(\gamma)\right)
$$

We suppose $\varepsilon(>0)$ is sufficiently small. Since any solution of $(2.1),(2.2)$ is translationinvariant, we can normalize $u$ by

$$
u(0)=\alpha,
$$

where $\alpha$ is an arbitrarily fixed number in the interval $\left(u_{-}(\gamma), u_{+}(\gamma)\right)$. We expect that a sharp transition layer appears in a neighborhood of $z=0$. Moreover, we put

$$
v(0)=\beta \in\left(v_{-}(\gamma), v_{+}(\gamma)\right),
$$

and $\beta$ will be determined later. 
First, we shall separate the whole interval $\mathbf{R}$ into two subintervals, say $\mathbf{R}_{-}$and $\mathbf{R}_{+}$, and consider the following boundary-value problems on each subinterval:

$$
\left.\begin{array}{c}
\varepsilon^{2}\left(u^{ \pm}\right)_{z z}-\varepsilon c \tau\left(u^{ \pm}\right)_{z}+f\left(u^{ \pm}, v^{ \pm}\right)=0 \\
\left(v^{ \pm}\right)_{z z}-c\left(v^{ \pm}\right)_{z}+g\left(u^{ \pm}, v^{ \pm} ; \gamma\right)=0
\end{array}\right\} \quad z \in \mathbf{R}_{ \pm}
$$

In $\S 2.1$, we construct outer solutions which approximate (2.5) outside of a boundary layer region. In $\S 2.2$, we construct inner solutions which approximate (2.5) in a boundary layer region. Second, in $\S 2.3$, using these approximate solutions we construct a uniformly approximate traveling wave solution of (2.1), (2.2), and (2.3), which gives us the information used to construct an exact solution. Finally, in $\S 2.4$, we define singular limit problems and look at the global pictures of these singular limit solutions.

2.1. Outer solutions. By putting $\varepsilon=0$ formally in (2.5), we get the following approximate problems:

$$
\begin{aligned}
& \left.\begin{array}{r}
f\left(u^{ \pm}, v^{ \pm}\right)=0 \\
\left(v^{ \pm}\right)_{z z}-c\left(v^{ \pm}\right)_{z}+g\left(u^{ \pm}, v^{ \pm} ; \gamma\right)=0
\end{array}\right\} \quad z \in \mathbf{R}_{ \pm}, \\
& v^{ \pm}( \pm \infty)=v_{ \pm}(\gamma), \quad v^{ \pm}(0)=\beta \text {. }
\end{aligned}
$$

We replace $f\left(u^{ \pm}, v^{ \pm}\right)=0$ by $u^{ \pm}=h_{ \pm}\left(v^{ \pm}\right)$. Thus, (2.6) can be reduced to

$$
\begin{aligned}
& \left(V^{ \pm}\right)_{z z}-c\left(V^{ \pm}\right)_{z}+g\left(h_{ \pm}\left(V^{ \pm}\right), V^{ \pm} ; \gamma\right)=0 \quad z \in \mathbf{R}_{ \pm}, \\
& V^{ \pm}( \pm \infty)=v_{ \pm}(\gamma), \quad V^{ \pm}(0)=\beta .
\end{aligned}
$$

Lemma 2.1. For any fixed $c \in \mathbf{R}, \beta \in\left(v_{-}(\gamma), v_{+}(\gamma)\right)$, and $\gamma \in\left(\gamma_{0}, \infty\right)$, there exist unique strictly monotone increasing solutions $V_{0}^{ \pm}(z ; c, \beta ; \gamma)\left(z \in \mathbf{R}_{ \pm}\right)$of $(2.7)$ satisfying

$$
\left|V_{0}^{ \pm}(z ; c, \beta ; \gamma)-v_{ \pm}(\gamma)\right| \in X_{\mu(c), 1}^{2}\left(\mathbf{R}_{ \pm}\right),
$$

where $\mu(c)=\min \left\{\mu_{-}(c), \mu_{+}(c)\right\}$ and $\mu_{ \pm}(c)$ are positive roots of

$$
\mu_{ \pm}^{2}-c \mu_{ \pm}+g_{u}\left(u_{ \pm}(\gamma), v_{ \pm}(\gamma) ; \gamma\right) h_{ \pm}^{\prime}\left(v_{ \pm}(\gamma)\right)+g_{v}\left(u_{ \pm}(\gamma), v_{ \pm}(\gamma) ; \gamma\right)=0
$$

Moreover $V_{0}^{ \pm}(z ; c, \beta ; \gamma)$ are continuous with respect to $(c, \beta) \in \mathbf{R} \times\left(v_{-}(\gamma), v_{+}(\gamma)\right)$ in the $X_{\mu(c), 1}^{2}\left(\mathbf{R}_{ \pm}\right)$-topology and satisfy

$$
\begin{aligned}
& \frac{\partial}{\partial \gamma}\left[\frac{d}{d z} V_{0}^{-}(0 ; c, \beta ; \gamma)-\frac{d}{d z} V_{0}^{+}(0 ; c, \beta ; \gamma)\right]>0 \\
& \frac{\partial}{\partial c}\left[\frac{d}{d z} V_{0}^{-}(0 ; c, \beta ; \gamma)-\frac{d}{d z} V_{0}^{+}(0 ; c, \beta ; \gamma)\right]>0
\end{aligned}
$$

and

$$
\frac{\partial}{\partial \beta}\left[\frac{d}{d z} V_{0}^{-}(0 ; c, \beta ; \gamma)-\frac{d}{d z} V_{0}^{+}(0 ; c, \beta ; \gamma)\right]>0
$$

From this lemma, we directly obtain the following result which will be useful in $\S 2.3$. 
Lemma 2.2. For any $c \in \mathbf{R}$ and $\gamma \in\left(\gamma_{0}, \infty\right)$, there exists a unique $\beta=\beta_{O}(c ; \gamma) \in$ $C^{1}(\mathbf{R})$ satisfying

$$
\frac{d}{d z} V_{0}^{-}\left(0 ; c, \beta_{O}(c ; \gamma) ; \gamma\right)-\frac{d}{d z} V_{0}^{+}\left(0 ; c, \beta_{O}(c ; \gamma) ; \gamma\right)=0
$$

The function $\beta_{O}(c ; \gamma)$ is strictly monotonically decreasing with respect to $c$ and $\gamma$ and converges to $v_{ \pm}(\gamma)$ as $c \rightarrow \mp \infty$, respectively. Moreover

$$
\gamma \lesseqgtr \gamma_{1} \quad \text { if and only if } \quad \beta_{O}(c ; \gamma) \gtreqless v^{*} .
$$

Remark 2.1. $\beta_{O}^{\prime}(c ; \gamma)$ is explicitly represented as

$$
\beta_{O}^{\prime}(c ; \gamma)=-\left.\frac{\int_{-\infty}^{0} e^{-c z}\left(\left(V_{0}^{-}\right)_{z}(z ; c, \beta ; \gamma)\right)^{2} d z+\int_{0}^{\infty} e^{-c z}\left(\left(V_{0}^{+}\right)_{z}(z ; c, \beta ; \gamma)\right)^{2} d z}{g\left(h_{+}(\beta), \beta ; \gamma\right)-g\left(h_{-}(\beta), \beta ; \gamma\right)}\right|_{\beta=\beta_{O}(c ; \gamma)},
$$

where ' means the derivative with respect to $c$. Furthermore, $\partial \beta_{O}(c ; \gamma) / \partial \gamma$ also is represented as

$$
\frac{\partial}{\partial \gamma} \beta_{O}(c ; \gamma)=-\left.\frac{\int_{-\infty}^{0} e^{-c z}\left(V_{0}^{-}\right)_{z}(z ; c, \beta ; \gamma) V_{0}^{-}(z ; c, \beta ; \gamma) d z}{+\int_{0}^{\infty} e^{-c z}\left(V_{0}^{+}\right)_{z}(z ; c, \beta ; \gamma) V_{0}^{+}(z ; c, \beta ; \gamma) d z}\right|_{\beta=\beta_{O}(c ; \gamma)}
$$

Using the functions $V_{0}^{ \pm}(z ; c, \beta ; \gamma)$, we define $U_{0}^{ \pm}(z ; c, \beta ; \gamma)$ by

$$
U_{0}^{ \pm}(z ; c, \beta ; \gamma)=h_{ \pm}\left(V_{0}^{ \pm}(z ; c, \beta ; \gamma)\right) \quad z \in \mathbf{R}_{ \pm} .
$$

We thus find that $\left(U_{0}^{ \pm}, V_{0}^{ \pm}\right)(z ; c, \beta ; \gamma)$ approximately satisfy $(2.5)$ outside of a neighborhood of $z=0$; however, $U_{0}^{ \pm}(z ; c, \beta ; \gamma)$ do not satisfy the boundary conditions $U_{0}^{ \pm}(0 ; c, \beta ; \gamma)=\alpha$.

2.2. Inner solutions. As stated above, $\left(U_{0}^{ \pm}, V_{0}^{ \pm}\right)(z ; c, \beta ; \gamma)$ does not satisfy (2.5) approximately in a neighborhood of $z=0$. That is, solutions $u^{ \pm}$of (2.5) have steep gradients. Therefore, we must remedy that defect by supplementing $\left(U_{0}^{ \pm}, V_{0}^{ \pm}\right)$with inner solutions $W_{0}^{ \pm}$in a neighborhood of $z=0$, so that $\left(U_{0}^{ \pm}+W_{0}^{ \pm}, V_{0}^{ \pm}\right)$will satisfy (2.5) approximately for all $z \in \mathbf{R}_{ \pm}$.

For this purpose, we introduce the stretched variable $\xi=z / \varepsilon$ in a neighborhood of $z=0$. Substituting $\left(U_{0}^{ \pm}+W_{0}^{ \pm}, V_{0}^{ \pm}\right)$into (2.5) and putting $\varepsilon=0$, we obtain the following approximate problems:

$$
\begin{aligned}
& \left(W_{0}^{ \pm}\right)_{\xi \xi}-c \tau\left(W_{0}^{ \pm}\right)_{\xi}+f\left(h_{ \pm}(\beta)+W_{0}^{ \pm}, \beta\right)=0 \quad \xi \in \mathbf{R}_{ \pm}, \\
& W_{0}^{ \pm}(0)=\alpha-h_{ \pm}(\beta), \\
& W_{0}^{ \pm}( \pm \infty)=0
\end{aligned}
$$

where $\beta$ is a fixed constant satisfying $\beta \in\left(v_{-}(\gamma), v_{+}(\gamma)\right)$. We first state the following lemma.

Lemma 2.3 (2). For any fixed $\beta \in\left[v_{-}(\gamma), v_{+}(\gamma)\right]$, consider the following problem:

$$
\begin{aligned}
& W_{\xi \xi}-c W_{\xi}+f(W, \beta)=0 \quad \xi \in \mathbf{R}, \\
& W( \pm \infty)=h_{ \pm}(\beta), \quad W(0)=\alpha .
\end{aligned}
$$

Then there exists $c=c_{0}(\beta) \in C^{1}\left(\left[v_{-}(\gamma), v_{+}(\gamma)\right]\right)$ such that (2.12) has a unique strictly monotone increasing solution $W(\xi ; \beta)$ satisfying

$$
\left|W(\xi ; \beta)-h_{ \pm}(\beta)\right| \in X_{\sigma_{ \pm}(\beta), 1}^{2}\left(\mathbf{R}_{ \pm}\right)
$$


where

$$
\sigma_{ \pm}(\beta)=\left[\mp c_{0}(\beta)+\left(\left(c_{0}(\beta)\right)^{2}-4 f_{u}\left(h_{ \pm}(\beta), \beta\right)\right)^{1 / 2}\right] / 2
$$

Furthermore,

$$
c_{0}(\beta) \lesseqgtr 0 \quad \text { if and only if } \quad \mathfrak{F}(\beta) \lesseqgtr 0 .
$$

Lemma 2.4. For any fixed $\beta \in\left[v_{-}(\gamma), v_{+}(\gamma)\right]$, let $c_{I}(\beta ; \tau)=c_{0}(\beta) / \tau$. Then there exists $\delta_{0}>0$ such that for any fixed $(\hat{c}, \hat{\beta}) \in \Lambda_{\delta_{0}} \equiv\left\{(\hat{c}, \hat{\beta})|| \hat{c}-c_{I}(\beta ; \tau)|+| \hat{\beta}-\beta \mid \leq\right.$ $\left.\delta_{0}\right\},(2.11)$ has unique strictly monotone increasing solutions $W_{0}^{ \pm}(\xi ; \hat{c}, \hat{\beta} ; \tau)$ satisfying

$$
\left|W_{0}^{ \pm}(\xi ; \hat{c}, \hat{\beta} ; \tau)\right| \in X_{\sigma_{ \pm}(\tau), 1}^{2}\left(\mathbf{R}_{ \pm}\right),
$$

where $\sigma_{ \pm}(\tau)=\inf _{(\hat{c}, \hat{\beta}) \in \Lambda_{\delta_{0}}} \sigma_{ \pm}(\hat{c}, \hat{\beta} ; \tau)$ and

$$
\sigma_{ \pm}(c, \beta ; \tau)=\left[\mp c \tau+\left((c \tau)^{2}-4 f_{u}\left(h_{ \pm}(\beta), \beta\right)\right)^{1 / 2}\right] / 2 .
$$

Furthermore, $W_{0}^{ \pm}(\xi ; \hat{c}, \hat{\beta} ; \tau)$ are continuous with respect to $(\hat{c}, \hat{\beta}) \in \Lambda_{\delta_{0}}$ in the $X_{\sigma_{ \pm}(\tau), 1}^{2}\left(\mathbf{R}_{ \pm}\right)$-topology and satisfy

$$
\begin{aligned}
\frac{d}{d \xi} W_{0}^{-}\left(0 ; c_{I}(\beta ; \tau), \beta ; \tau\right)-\frac{d}{d \xi} W_{0}^{+}\left(0 ; c_{I}(\beta ; \tau), \beta ; \tau\right) & =0 \\
\frac{\partial}{\partial c}\left[\frac{d}{d \xi} W_{0}^{-}\left(0 ; c_{I}(\beta ; \tau), \beta ; \tau\right)-\frac{d}{d \xi} W_{0}^{+}\left(0 ; c_{I}(\beta ; \tau), \beta ; \tau\right)\right] & >0 \\
\frac{\partial}{\partial \beta}\left[\frac{d}{d \xi} W_{0}^{-}\left(0 ; c_{I}(\beta ; \tau), \beta ; \tau\right)-\frac{d}{d \xi} W_{0}^{+}\left(0 ; c_{I}(\beta ; \tau), \beta ; \tau\right)\right] & >0 .
\end{aligned}
$$

Remark 2.2. It follows from (2.13), (2.14), and (2.15) that $\frac{d}{d \beta} c_{I}(\beta ; \tau)$ is strictly negative for $\beta \in\left[v_{-}(\gamma), v_{+}(\gamma)\right]$. Then there exists an inverse function of $c=c_{I}(\beta ; \tau)$, say $\beta=\beta_{I}(c ; \tau)$, which is strictly decreasing for $c \in\left[c_{I}\left(v_{+}(\gamma) ; \tau\right), c_{I}\left(v_{-}(\gamma) ; \tau\right)\right]$.

2.3. Uniformly approximate solutions. In the preceding subsections, we constructed the lowest-order approximations $\left(U_{0}^{ \pm}(z ; c, \beta ; \gamma)+W_{0}^{ \pm}(z / \varepsilon ; c, \beta ; \tau)\right.$, $\left.V_{0}^{ \pm}(z ; c, \beta ; \gamma)\right)$ of the problems $(2.5)$. It is clear that these approximations are matched at $z=0$ in the $C^{0}$-sense. In order for these to be approximations of $(2.1),(2.2)$, and (2.3) uniformly on $\mathbf{R}$, their derivatives have to be matched at $z=0$ in the $C^{0}$-sense. That is, we impose the following conditions on $\left(W_{0}^{ \pm}, V_{0}^{ \pm}\right)$:

$$
\begin{aligned}
& \Phi_{0}(c, \beta ; \tau) \equiv \frac{d}{d \xi} W_{0}^{-}(0 ; c, \beta ; \tau)-\frac{d}{d \xi} W_{0}^{+}(0 ; c, \beta ; \tau)=0 \\
& \Psi_{0}(c, \beta ; \gamma) \equiv \frac{d}{d z} V_{0}^{-}(0 ; c, \beta ; \gamma)-\frac{d}{d z} V_{0}^{+}(0 ; c, \beta ; \gamma)=0 .
\end{aligned}
$$

From Lemmas 2.2 and 2.4, it follows that the above relations are equivalent to the conditions

$$
c=c_{0}(\beta) / \tau
$$

and

$$
\beta=\beta_{O}(c ; \gamma)
$$

respectively. We note that by Remark $2.2,(2.17)$ is identical to

$$
\beta=\beta_{I}(c ; \tau) \text {. }
$$


Lemma 2.5. When $\gamma_{0}<\gamma<\gamma_{2}$, there exists $\tau_{c}(\gamma)$ such that the curves (2.18) and (2.19) (or (2.17)) have three intersection points for $0<\tau<\tau_{c}(\gamma)$, two for $\tau=\tau_{c}(\gamma)$, and one for $\tau>\tau_{c}(\gamma)$. When $\gamma \geq \gamma_{2}$, the curves (2.18) and (2.19) have only one intersection point for each $\tau$. Furthermore, these curves intersect transversally at each point except when there is only one point in the case of $\gamma_{0}<\gamma<\gamma_{2}$ and $\tau=\tau_{c}(\gamma)$ (see Figure 4).

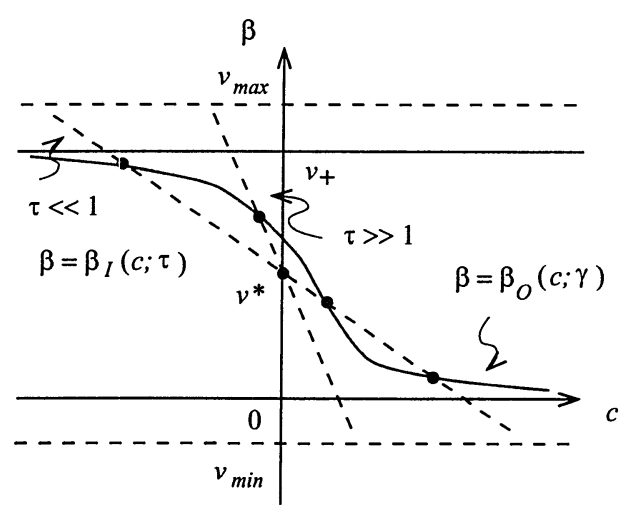

(a)

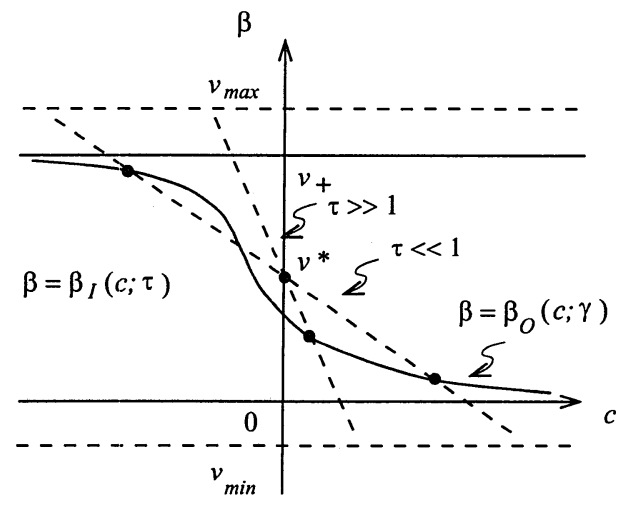

(c)

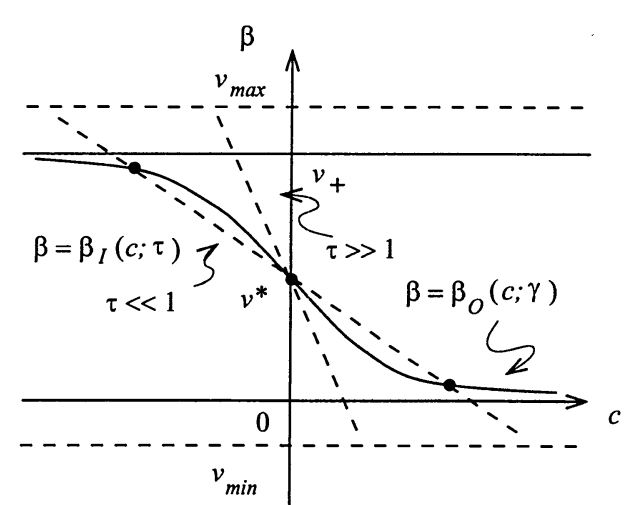

(b)

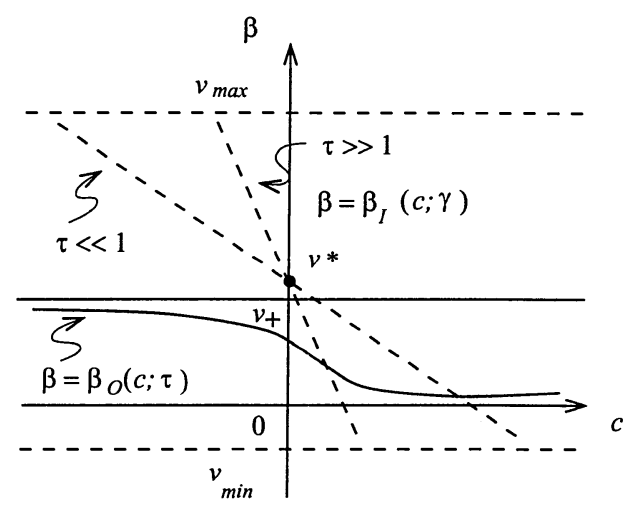

(d)

FIGURE 4. Intersection of the curves $\beta=\beta_{O}(c ; \gamma)$ and $\beta=\beta_{I}(c ; \tau)$ for different values of $\gamma$ : (a) $\gamma_{0}<\gamma<\gamma_{1}$, (b) $\gamma=\gamma_{1}$, (c) $\gamma_{1}<\gamma<\gamma_{2}$, (d) $\gamma \geq \gamma_{2}$.

Let $\left(c_{f}(\tau, \gamma), \beta_{f}(\tau, \gamma)\right)$ be an arbitrary intersection point of the curves $(2.18)$ and (2.19). Define

$$
u_{0}^{f}(z ; \varepsilon ; \tau, \gamma)= \begin{cases}U_{0}^{-}\left(z ; c_{f}(\tau, \gamma), \beta_{f}(\tau, \gamma) ; \gamma\right)+W_{0}^{-}\left(z / \varepsilon ; c_{f}(\tau, \gamma) \beta_{f}(\tau, \gamma) ; \tau\right) & z \in \mathbf{R}_{-} \\ U_{0}^{+}\left(z ; c_{f}(\tau, \gamma), \beta_{f}(\tau, \gamma) ; \gamma\right)+W_{0}^{+}\left(z / \varepsilon ; c_{f}(\tau, \gamma) \beta_{f}(\tau, \gamma) ; \tau\right) & z \in \mathbf{R}_{+}\end{cases}
$$

and

$$
v_{0}^{f}(z ; \varepsilon ; \tau, \gamma)= \begin{cases}V_{0}^{-}\left(z ; c_{f}(\tau, \gamma), \beta_{f}(\tau, \gamma) ; \gamma\right) & z \in \mathbf{R}_{-} \\ V_{0}^{+}\left(z ; c_{f}(\tau, \gamma), \beta_{f}(\tau, \gamma) ; \gamma\right) & z \in \mathbf{R}_{+}\end{cases}
$$


Then, we find that $\left(u_{0}^{f}, v_{0}^{f}\right)(z ; \varepsilon ; \tau, \gamma)$ is the lowest-order uniform approximation on $\mathbf{R}$ to the problem (2.1), (2.2), and (2.3). From Lemma 2.5, we see that the number of uniformly approximate traveling wave solutions depends on $\tau$ and $\gamma$.

Lemma 2.6. When $\gamma_{0}<\gamma<\gamma_{2},(2.1),(2.2)$, and (2.3) has three uniformly approximate traveling front waves $\left(u_{0}^{f}, v_{0}^{f}\right)(z ; \varepsilon ; \tau, \gamma)$ with velocity $c=c_{f}(\tau, \gamma)$ for $0<\tau<$ $\tau_{c}(\gamma)$, two for $\tau=\tau_{c}(\gamma)$ and one for $\tau>\tau_{c}(\gamma)$. On the other hand, when $\gamma \geq \gamma_{2}$, it has only one for any $\tau$ (see Figure 5).

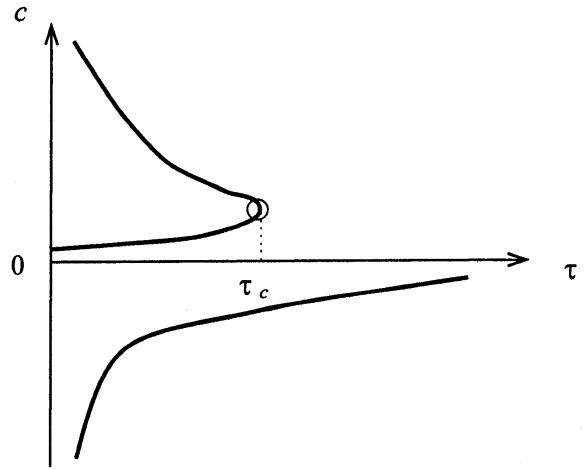

(a)

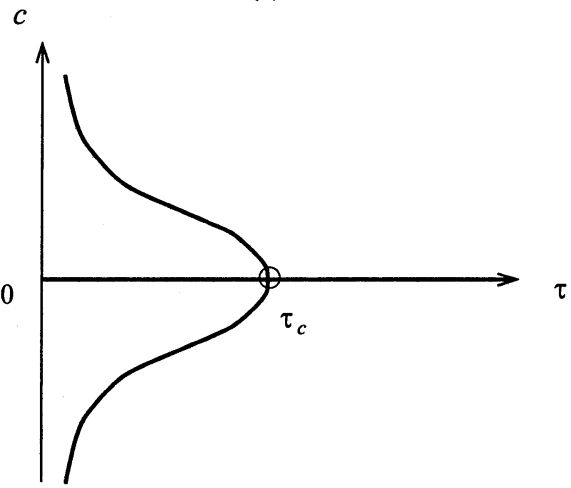

(b)

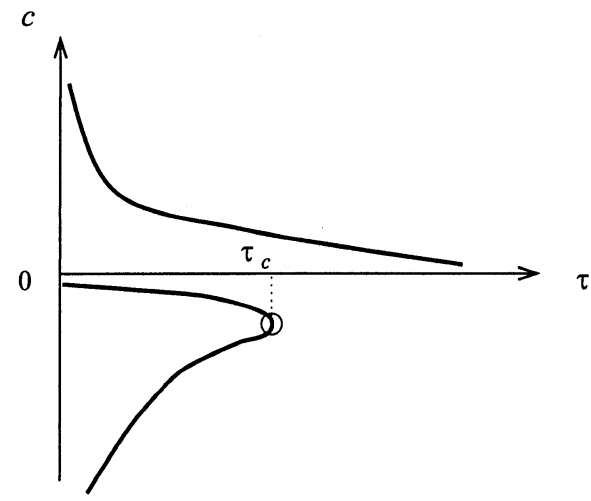

(c)

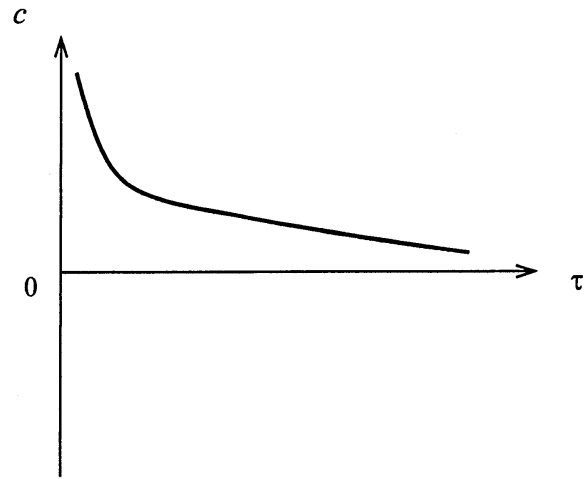

(d)

FIgURE 5. Singular limit velocity curves $c$ versus $\tau$ for different values of $\gamma$ : (a) $\gamma_{0}<\gamma<\gamma_{1}$, (b) $\gamma=\gamma_{1}$, (c) $\gamma_{1}<\gamma<\gamma_{2}$, (d) $\gamma \geq \gamma_{2}$.

If $\left(c_{f}(\tau, \gamma), \beta_{f}(\tau, \gamma)\right)$ is an arbitrary intersection point of the curves (2.18) and (2.19) at which these intersect transversally, that is,

$$
\beta_{I}^{\prime}(c ; \tau)-\left.\beta_{O}^{\prime}(c ; \gamma)\right|_{c=c_{f}(\tau, \gamma)} \neq 0
$$

is satisfied, then by using the implicit function theorem, we can find an exact solution of (2.1), (2.2), and (2.3) in a neighborhood of $\left(u_{0}^{f}, v_{0}^{f}\right)(z ; \varepsilon ; \tau, \gamma)$ (see [5]).

Theorem 2.1. When $\gamma_{0}<\gamma<\gamma_{2}$, (2.1), (2.2), and (2.3) has three traveling front waves $\left(u^{f}, v^{f}\right)(z ; \varepsilon ; \tau, \gamma)$ with velocity $c=c_{f}(\varepsilon ; \tau, \gamma)$ for $0<\tau<\tau_{c}(\gamma)$, and one for $\tau \geq \tau_{c}(\gamma)$. On the other hand, when $\gamma \geq \gamma_{2}$, it has only one for any $\tau$, where $\left(u^{f}, v^{f}\right)(z ; \varepsilon ; \tau, \gamma)$ and $c_{f}(\varepsilon ; \tau, \gamma)$ satisfy

$$
\left\|u^{f}(\cdot ; \varepsilon ; \tau, \gamma)-u_{0}^{f}(\cdot ; \varepsilon ; \tau, \gamma)\right\|_{X_{\rho, \varepsilon}^{1}(\mathbf{R})}+\left\|v^{f}(\cdot ; \varepsilon ; \tau, \gamma)-v_{0}^{f}(\cdot ; \varepsilon ; \tau, \gamma)\right\|_{X_{\rho, \varepsilon}^{1}(\mathbf{R})} \longrightarrow 0
$$


and

$$
c_{f}(\varepsilon ; \tau, \gamma) \longrightarrow c_{f}(\tau, \gamma)
$$

as $\varepsilon \longrightarrow 0$.

As for the stability property of these traveling front waves, it is shown in [7] that the upper and lower branches are stable and the middle one is unstable (see Figure $5)$. More precisely, the sign of the left-hand side of (2.20) corresponds one-to-one to the stability property of a traveling front wave. Namely, this is equivalent to the sign of the real part of the principal eigenvalue of the linearized eigenvalue problem.

2.4. Singular limit problems for traveling front and back waves. We presented a method to construct traveling wave solutions with the aid of outer and inner solutions, that is, a usual singular perturbation method as in [5]. Now, let us review our method. What are the simplest limiting equations of (2.1), (2.2), and (2.3) that hold important information about the existence and the stability property of an exact solution? Formally, putting $\varepsilon=0$ in (2.1), we obtained the problems (2.7), which describe the outer solutions. However, these are not sufficient for our purpose. Then, we had to consider the other problems (2.11), which describe the inner solutions. If we impose the relation $\beta=\beta_{I}(c ; \tau)$ in advance, the inner solutions $W_{0}^{ \pm}(\xi ; c, \beta ; \tau)$ of $(2.11)$ are matched at $\xi=0$ in the $C^{1}$-sense automatically. Thus, combining the relation $\beta=\beta_{I}(c ; \tau)$ with (2.7), we can obtain the following limiting equations:

$$
\begin{aligned}
& \left(V^{ \pm}\right)_{z z}-c\left(V^{ \pm}\right)_{z}+g\left(h_{ \pm}\left(V^{ \pm}\right), V^{ \pm} ; \gamma\right)=0 \quad z \in \mathbf{R}_{ \pm} \\
& V^{ \pm}( \pm \infty)=v_{ \pm}(\gamma), \quad V^{ \pm}(0)=\beta_{I}(c ; \tau) .
\end{aligned}
$$

By Lemma 2.1, we can find solutions $V_{f}^{ \pm}(z ; c ; \tau, \gamma)$ of $(2.21)$. Furthermore, we impose the relation

$$
\left(V_{f}^{-}\right)_{z}(0 ; c ; \tau, \gamma)=\left(V_{f}^{+}\right)_{z}(0 ; c ; \tau, \gamma)
$$

which is equivalent to the condition $\beta_{I}(c ; \tau)=\beta_{O}(c ; \gamma)$. From (2.22), we can determine the relation $c=c_{f}(\tau, \gamma)$, which is the same velocity as determined in $\S 2.3$. (2.21) and (2.22) are the simplest limiting problem to (2.1), (2.2), and (2.3), because if we get solutions $V_{f}^{ \pm}\left(z ; c_{f}(\tau, \gamma) ; \tau, \gamma\right)$ satisfying $(2.21)$ and $(2.22)$, we can find a uniformly approximate solution $\left(u_{0}^{f}, v_{0}^{f}\right)(z ; \varepsilon ; \tau, \gamma)$ of (2.1), (2.2), and (2.3) as follows:

$$
\begin{aligned}
& u_{0}^{f}(z ; \varepsilon ; \tau, \gamma) \\
& \quad= \begin{cases}h_{-}\left(V_{f}^{-}\left(z ; c_{f}(\tau, \gamma) ; \tau, \gamma\right)\right)+W_{0}^{-}\left(z / \varepsilon ; c_{f}(\tau, \gamma), \beta_{I}\left(c_{f}(\tau, \gamma), \tau\right) ; \tau\right) & z \in \mathbf{R}_{-}, \\
h_{+}\left(V_{f}^{+}\left(z ; c_{f}(\tau, \gamma) ; \tau, \gamma\right)\right)+W_{0}^{+}\left(z / \varepsilon ; c_{f}(\tau, \gamma), \beta_{I}\left(c_{f}(\tau, \gamma), \tau\right) ; \tau\right) & z \in \mathbf{R}_{+},\end{cases}
\end{aligned}
$$

and

$$
v_{0}^{f}(z ; \varepsilon ; \tau, \gamma)= \begin{cases}V_{f}^{-}\left(z ; c_{f}(\tau, \gamma) ; \tau, \gamma\right) & z \in \mathbf{R}_{-} \\ V_{f}^{+}\left(z ; c_{f}(\tau, \gamma) ; \tau, \gamma\right) & z \in \mathbf{R}_{+}\end{cases}
$$

Then we call (2.21) and (2.22) a singular limit problem for traveling front waves and call $\left(u_{0}^{f}, v_{0}^{f}\right)(z ; \varepsilon ; \tau, \gamma)(z \in \mathbf{R})$ and $c_{f}(\tau, \gamma)$ a singular limit traveling front wave and a singular limit velocity, respectively. We write a singular limit traveling front wave as $P \rightarrow Q$ symbolically. 
An important condition to get an exact solution from a uniformly approximate solution, say $(2.20)$, is

$$
\frac{\partial}{\partial c}\left(V_{f}^{-}\right)_{z}(0 ; c ; \tau, \gamma)-\left.\frac{\partial}{\partial c}\left(V_{f}^{+}\right)_{z}(0 ; c ; \tau, \gamma)\right|_{c=c_{f}(\tau, \gamma)} \neq 0
$$

for the singular limit problem (2.21) and (2.22). Furthermore, noting the following relation between the left-hand sides of $(2.20)$ and (2.23):

$$
\begin{gathered}
\frac{\partial}{\partial c}\left(V_{f}^{-}\right)_{z}(0 ; c ; \tau, \gamma)-\frac{\partial}{\partial c}\left(V_{f}^{+}\right)_{z}(0 ; c ; \tau, \gamma)=\frac{d}{d c} \Psi_{0}\left(c, \beta_{I}(c ; \tau) ; \gamma\right) \\
=\frac{\partial}{\partial c} \Psi_{0}\left(c, \beta_{I}(c ; \tau) ; \gamma\right)+\frac{\partial}{\partial \beta} \Psi_{0}\left(c, \beta_{I}(c ; \tau) ; \gamma\right) \cdot \beta_{I}^{\prime}(c ; \tau) \\
=\frac{\partial}{\partial \beta} \Psi_{0}\left(c, \beta_{I}(c ; \tau) ; \gamma\right) \cdot\left\{\beta_{I}^{\prime}(c ; \tau)-\beta_{O}^{\prime}(c ; \gamma)\right\}
\end{gathered}
$$

where $\frac{\partial}{\partial \beta} \Psi_{0}\left(c, \beta_{I}(c ; \tau) ; \gamma\right)>0$, we find that the sign of the left-hand side of $(2.23)$ determines the stability property of an exact solution.

Similarly, we can consider a singular limit problem of traveling back waves:

$$
\begin{aligned}
& \left(V^{ \pm}\right)_{z z}-c\left(V^{ \pm}\right)_{z}+g\left(h_{ \pm}\left(V^{ \pm}\right), V^{ \pm} ; \gamma\right)=0 \quad z \in \mathbf{R}_{ \pm}, \\
& V^{ \pm}( \pm \infty)=v_{\mp}(\gamma), \quad V^{ \pm}(0)=\beta_{I}(-c ; \tau),
\end{aligned}
$$

and

$$
\left(V^{-}\right)_{z}(0 ; c ; \tau, \gamma)=\left(V^{+}\right)_{z}(0 ; c ; \tau, \gamma)
$$

Let $V_{b}^{ \pm}(z ; c ; \tau, \gamma)\left(z \in \mathbf{R}_{ \pm}\right)$be solutions of $(2.25),(2.26)$ and $W_{0}^{ \pm}(\xi ; c, \beta ; \tau)\left(\xi \in \mathbf{R}_{ \pm}\right)$ be solutions of (2.11). Put

$$
\begin{aligned}
& u_{0}^{b}(z ; \varepsilon ; \tau, \gamma) \\
& =\left\{\begin{array}{ll}
h_{-}\left(V_{b}^{-}\left(z ; c_{b}(\tau, \gamma) ; \tau, \gamma\right)\right)+W_{0}^{-}\left(-z / \varepsilon ; c_{b}(\tau, \gamma), \beta_{I}\left(c_{b}(\tau, \gamma), \tau\right) ; \tau\right) & z \in \mathbf{R}_{-}, \\
h_{+}\left(V_{b}^{+}\left(z ; c_{b}(\tau, \gamma) ; \tau, \gamma\right)\right)+W_{0}^{+}\left(-z / \varepsilon ; c_{b}(\tau, \gamma), \beta_{I}\left(c_{b}(\tau, \gamma), \tau\right) ; \tau\right) & z \in \mathbf{R}_{+},
\end{array},\right.
\end{aligned}
$$

and

$$
v_{0}^{b}(z ; \varepsilon ; \tau, \gamma)= \begin{cases}V_{b}^{-}\left(z ; c_{b}(\tau, \gamma) ; \tau, \gamma\right) & z \in \mathbf{R}_{-} \\ V_{b}^{+}\left(z ; c_{b}(\tau, \gamma) ; \tau, \gamma\right) & z \in \mathbf{R}_{+}\end{cases}
$$

where $V_{b}^{ \pm}(z ; c ; \tau, \gamma)=V_{f}^{ \pm}(-z ;-c ; \tau, \gamma)$ and $c_{b}(\tau, \gamma)=-c_{f}(\tau, \gamma)$. Then, $\left(u_{0}^{b}, v_{0}^{b}\right)$ $(z ; \varepsilon ; \tau, \gamma)$ will be a uniformly approximate solution of $(2.1),(2.2)^{\prime}$, and $(2.3)$, where

$$
(u, v)(-\infty)=Q \equiv\left(u_{+}(\gamma), v_{+}(\gamma)\right), \quad(u, v)(+\infty)=P \equiv\left(u_{-}(\gamma), v_{-}(\gamma)\right)
$$

We call a solution $\left(u_{0}^{b}, v_{0}^{b}\right)(z ; \varepsilon ; \tau, \gamma)(z \in \mathbf{R})$ and $c_{b}(\tau, \gamma)$ a singular limit traveling back wave and a singular limit velocity, respectively. Similarly, we write a singular limit traveling back wave as $Q \rightarrow P$ symbolically.

Finally, regarding $\gamma$ as a bifurcation parameter, we give the bifurcation diagrams for singular limit front waves $P \rightarrow Q, c=c_{f}(\tau, \gamma)$, and back waves $Q \rightarrow P, c=c_{b}(\tau, \gamma)$, in Figure 6 for any fixed $\tau$. These pictures play an essential part in the next section. 


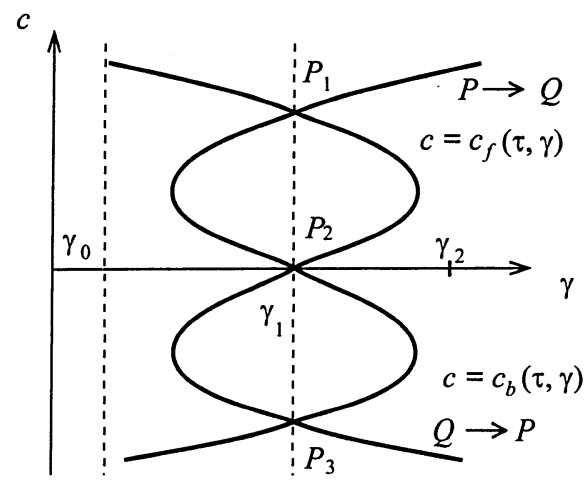

(a)

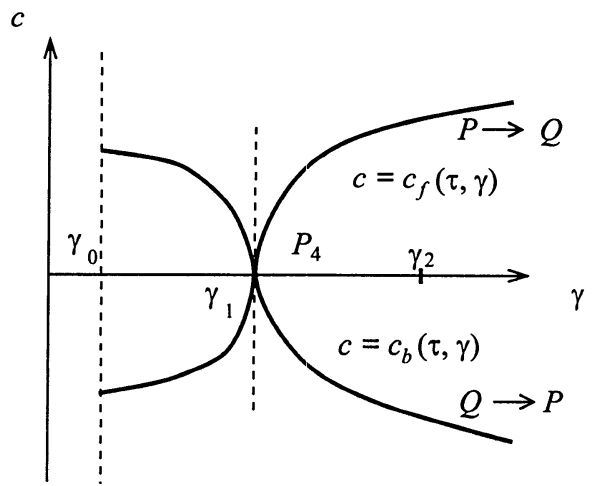

(b)

$c$

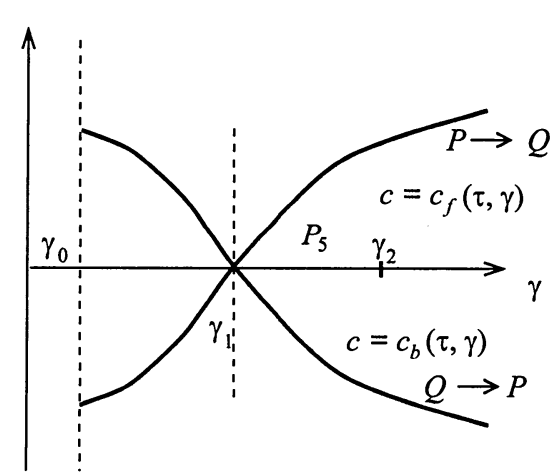

(c)

FiguRE 6. Singular limit velocity curves $c$ versus $\gamma$ for different values of $\tau$ : (a) $0<\tau<\tau_{c}\left(\gamma_{1}\right)$, (b) $\tau=\tau_{c}\left(\gamma_{1}\right)$, (c) $\tau>\tau_{c}\left(\gamma_{1}\right)$.

\section{Singular pulse waves bifurcating from front and back waves}

We will show a local bifurcation structure of traveling pulse waves in the $(c, \gamma)$ parameter space near the intersection points of the two curves of parameters corresponding to the singular limit traveling front and back waves. At the points $P_{i}$ $(i=1,2,3,4,5)$ in Figure 6 , traveling front waves $P \rightarrow Q$ and back waves $Q \rightarrow P$ with the same velocities coexist. This situation suggests that traveling pulse-like waves connecting $P$ at $z=-\infty$ with $P$ at $z=\infty$ exist, with infinitely long excited regions. This suggests the possibility of the existence of traveling pulse waves, with arbitrarily long (but finite) excited regions in some neighborhood of $\gamma_{1}$ (see Figure 7).

Our purpose is to construct a traveling pulse wave of the problem

$$
\left.\begin{array}{rl}
\varepsilon^{2} u_{z z}-\varepsilon c \tau u_{z}+f(u, v) & =0 \\
v_{z z}-c v_{z}+g(u, v ; \gamma) & =0
\end{array}\right\} \quad z \in \mathbf{R}
$$

with boundary conditions

$$
(u, v)( \pm \infty)=P \equiv\left(u_{-}(\gamma), v_{-}(\gamma)\right)
$$

or

$$
(u, v)( \pm \infty)=Q \equiv\left(u_{+}(\gamma), v_{+}(\gamma)\right)
$$




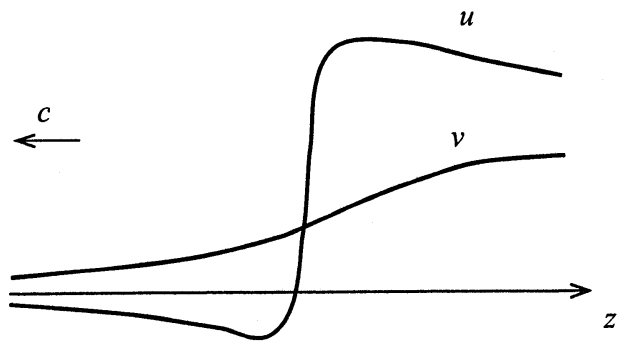

(a)

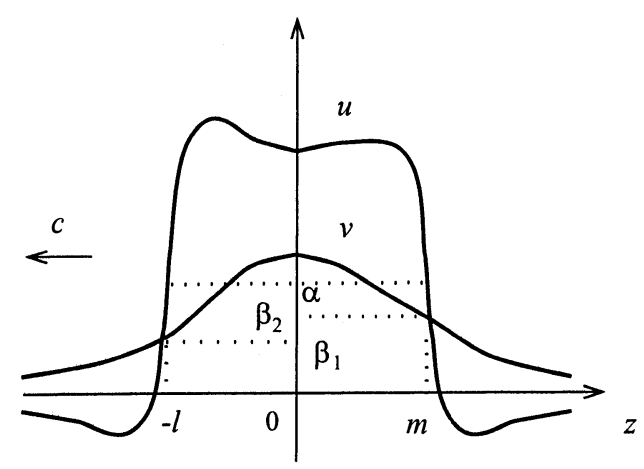

(c)

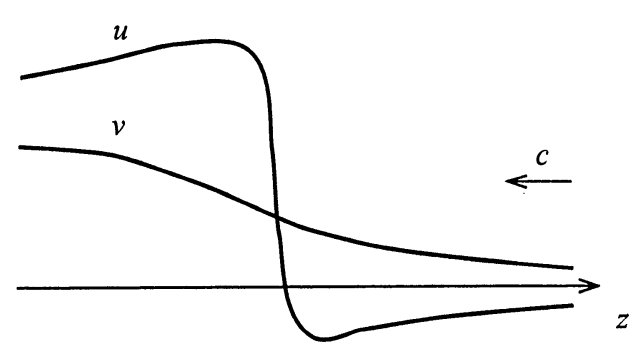

(b)

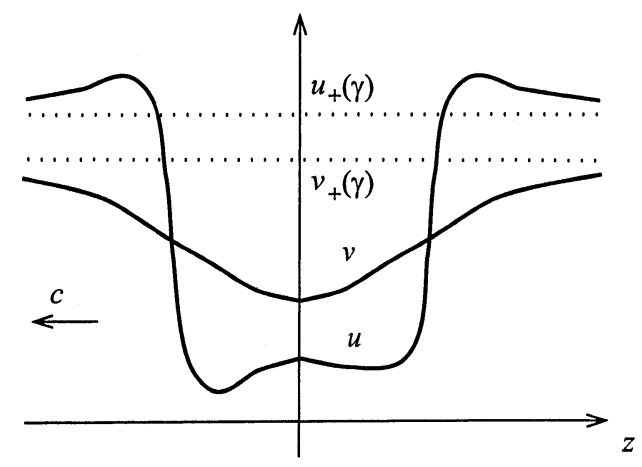

(d)

Figure 7. Shapes of front, back, $P$-pulse and $Q$-pulse waves: (a) front wave, (b) back wave, $(\mathrm{c})$ front + back wave $(P$-pulse wave $),(d)$ back + front wave $(Q$-pulse wave).

for $\gamma$ close to $\gamma_{1}$ with the aid of traveling front and back waves. We call a solution satisfying (3.1), (3.2) or (3.1), (3.3) a $P$-pulse wave or a $Q$-pulse wave, and write it symbolically as $P \rightarrow P$ or $Q \rightarrow Q$, respectively. We restrict our attention to the case where we find a $P$-pulse wave of the problem (3.1), (3.2) for $(c, \gamma)$ in a neighborhood of $P_{1}$. We comment on other cases later. We suppose that this solution has two sharp transition layers at $z=-\ell$ and $z=m$ (see Figure $7(\mathrm{c}))$. Normalize $(u, v)(z ; \varepsilon ; \tau, \gamma)$ to satisfy

$$
v_{z}(0 ; \varepsilon ; \tau, \gamma)=0
$$

and define $\nu, \ell, m, \beta_{1}$, and $\beta_{2}$ such that

$$
\begin{gathered}
u(0 ; \varepsilon ; \tau, \gamma)=\nu, \quad u(-\ell ; \varepsilon ; \tau, \gamma)=\alpha, \quad u(m ; \varepsilon ; \tau, \gamma)=\alpha \\
v(-\ell ; \varepsilon ; \tau, \gamma)=\beta_{1}, \quad v(m ; \varepsilon ; \tau, \gamma)=\beta_{2},
\end{gathered}
$$

where $\alpha$ is arbitrarily fixed in the interval $\left(u_{-}(\gamma), u_{+}(\gamma)\right)$. Then, we can separate the whole line $\mathbf{R}$ into four subintervals and consider the following problems:

$$
\begin{aligned}
& \left.\begin{array}{rl}
\varepsilon^{2} u_{z z}-\varepsilon c \tau u_{z}+f(u, v) & =0 \\
v_{z z}-c v_{z}+g(u, v ; \gamma) & =0
\end{array}\right\} \quad z \in(-\infty,-\ell) \\
& u(-\infty)=u_{-}(\gamma), \quad u(-\ell)=\alpha, \\
& v(-\infty)=v_{-}(\gamma), \quad v(-\ell)=\beta_{1},
\end{aligned}
$$




$$
\left.\begin{array}{c}
\varepsilon^{2} u_{z z}-\varepsilon c \tau u_{z}+f(u, v)=0 \\
v_{z z}-c v_{z}+g(u, v ; \gamma)=0
\end{array}\right\} \quad z \in(-\ell, 0),
$$

respectively. The standard classical singular perturbation process is as follows: first, solve (3.5a)-(3.5d) independently and examine the dependency of the parameters $c$, $\nu, \ell, m, \beta_{1}, \beta_{2}$ on each solution. Second, match the solutions smoothly at the points $z=-\ell, z=0$ and $z=m$ and then determine the parameters $c, \nu, \ell, m, \beta_{1}, \beta_{2}$ as functions of $\varepsilon, \tau$, and $\gamma$. But in this section, we do not follow this process. Instead, we consider a singular limit problem (3.1), (3.2). Formally, putting $\varepsilon=0$ in (3.5a)-(3.5d), we have

$$
\begin{aligned}
& \left(V^{(1)}\right)_{z z}-c\left(V^{(1)}\right)_{z}+g\left(h_{-}\left(V^{(1)}\right), V^{(1)} ; \gamma\right)=0 \quad z \in(-\infty,-\ell), \\
& V^{(1)}(-\infty)=v_{-}(\gamma), \quad V^{(1)}(-\ell)=\beta_{1} \text {, } \\
& \left(V^{(2)}\right)_{z z}-c\left(V^{(2)}\right)_{z}+g\left(h_{+}\left(V^{(2)}\right), V^{(2)} ; \gamma\right)=0 \quad z \in(-\ell, 0) \\
& V^{(2)}(-\ell)=\beta_{1}, \quad\left(V^{(2)}\right)_{z}(0)=0 \text {, } \\
& \left(V^{(3)}\right)_{z z}-c\left(V^{(3)}\right)_{z}+g\left(h_{+}\left(V^{(3)}\right), V^{(3)} ; \gamma\right)=0 \quad z \in(0, m), \\
& \left(V^{(3)}\right)_{z}(0)=0, \quad V^{(3)}(m)=\beta_{2} \text {, } \\
& \left(V^{(4)}\right)_{z z}-c\left(V^{(4)}\right)_{z}+g\left(h_{-}\left(V^{(4)}\right), V^{(4)} ; \gamma\right)=0 \quad z \in(m, \infty) \\
& V^{(4)}(m)=\beta_{2}, \quad V^{(4)}(\infty)=v_{-}(\gamma)
\end{aligned}
$$

respectively. The component $u$ of a solution satisfying (3.1), (3.2) has two internal transition layers at $z=-\ell$ and $m$ (see Figure $7(\mathrm{c})$ ). Then introducing the stretched variable $\xi=(z+\ell) / \varepsilon$ in a neighborhood of $z=-\ell$ and putting $\varepsilon=0$, (3.5a) and (3.5b) become

$$
\begin{aligned}
& \left(W_{1}^{ \pm}\right)_{\xi \xi}-c \tau\left(W_{1}^{ \pm}\right)_{\xi}+f\left(h_{ \pm}\left(\beta_{1}\right)+W_{1}^{ \pm}, \beta_{1}\right)=0 \quad \xi \in \mathbf{R}_{ \pm} \\
& W_{1}^{ \pm}(0)=\alpha-h_{ \pm}\left(\beta_{1}\right) \\
& W_{1}^{ \pm}( \pm \infty)=0
\end{aligned}
$$


Similarly, by using $\xi=(z-m) / \varepsilon$ in a neighborhood of $z=m,(3.6 \mathrm{c})$ and (3.6d) become

$$
\begin{aligned}
& \left(W_{2}^{ \pm}\right)_{\xi \xi}-c \tau\left(W_{2}^{ \pm}\right)_{\xi}+f\left(h_{ \pm}\left(\beta_{2}\right)+W_{2}^{ \pm}, \beta_{2}\right)=0, \quad \xi \in \mathbf{R}_{ \pm} \\
& W_{2}^{ \pm}(0)=\alpha-h_{ \pm}\left(\beta_{2}\right), \\
& W_{2}^{ \pm}( \pm \infty)=0 .
\end{aligned}
$$

In order to obtain smooth solutions on $\mathbf{R}$ of the problems (3.7), (3.8), we must impose the conditions

$$
\beta_{1}=\beta_{I}(c ; \tau), \quad \beta_{2}=\beta_{I}(-c ; \tau),
$$

respectively (see Lemma 2.4 and Remark 2.2). Therefore, substituting (3.9) into (3.6a)-(3.6d), we have the singular limit problem of (3.1), (3.2):

$$
\begin{aligned}
& \left(V^{(1)}\right)_{z z}-c\left(V^{(1)}\right)_{z}+g\left(h_{-}\left(V^{(1)}\right), V^{(1)} ; \gamma\right)=0 \quad z \in(-\infty,-\ell), \\
& V^{(1)}(-\infty)=v_{-}(\gamma), \quad V^{(1)}(-\ell)=\beta_{I}(c ; \tau) \text {, } \\
& \left(V^{(2)}\right)_{z z}-c\left(V^{(2)}\right)_{z}+g\left(h_{+}\left(V^{(2)}\right), V^{(2)} ; \gamma\right)=0 \quad z \in(-\ell, 0), \\
& V^{(2)}(-\ell)=\beta_{I}(c ; \tau), \quad\left(V^{(2)}\right)_{z}(0)=0 \text {, } \\
& \left(V^{(3)}\right)_{z z}-c\left(V^{(3)}\right)_{z}+g\left(h_{+}\left(V^{(3)}\right), V^{(3)} ; \gamma\right)=0 \quad z \in(0, m) \\
& \left(V^{(3)}\right)_{z}(0)=0, \quad V^{(3)}(m)=\beta_{I}(-c ; \tau) \text {, } \\
& \left(V^{(4)}\right)_{z z}-c\left(V^{(4)}\right)_{z}+g\left(h_{-}\left(V^{(4)}\right), V^{(4)} ; \gamma\right)=0 \quad z \in(m, \infty), \\
& V^{(4)}(m)=\beta_{I}(-c ; \tau), \quad V^{(4)}(\infty)=v_{-}(\gamma) \text {, } \\
& \left(V^{(1)}\right)_{z}(-\ell)=\left(V^{(2)}\right)_{z}(-\ell) \\
& \left(V^{(2)}\right)(0)=\left(V^{(3)}\right)(0), \\
& \left(V^{(3)}\right)_{z}(m)=\left(V^{(4)}\right)_{z}(m) \text {. }
\end{aligned}
$$

We want to apply phase-plane methods to find solutions of the problems (3.10a)(3.10d), (3.11). To do so, we rewrite them as the following equivalent first-order systems:

$$
\begin{aligned}
& \left.\begin{array}{l}
\left(V^{(1)}\right)_{z}=Y^{(1)} \\
\left(Y^{(1)}\right)_{z}=c Y^{(1)}-g\left(h_{-}\left(V^{(1)}\right), V^{(1)} ; \gamma\right)
\end{array}\right\} \quad z \in(-\infty,-\ell), \\
& V^{(1)}(-\infty)=v_{-}(\gamma), \quad Y^{(1)}(-\infty)=0, \\
& V^{(1)}(-\ell)=\beta_{I}(c ; \tau) \text {, } \\
& \left.\begin{array}{l}
\left(V^{(2)}\right)_{z}=Y^{(2)} \\
\left(Y^{(2)}\right)_{z}=c Y^{(2)}-g\left(h_{+}\left(V^{(2)}\right), V^{(2)} ; \gamma\right)
\end{array}\right\} \quad z \in(-\ell, 0), \\
& V^{(2)}(-\ell)=\beta_{I}(c ; \tau), \quad Y^{(2)}(-\ell)=Y^{(1)}(-\ell), \\
& \left(V^{(2)}\right)_{z}(0)=0
\end{aligned}
$$




$$
\begin{aligned}
& \left.\begin{array}{l}
\left(V^{(3)}\right)_{z}=Y^{(3)} \\
\left(Y^{(3)}\right)_{z}=c Y^{(3)}-g\left(h_{+}\left(V^{(3)}\right), V^{(3)} ; \gamma\right)
\end{array}\right\} \quad z \in(0, m), \\
& V^{(3)}(m)=\beta_{I}(-c ; \tau), \quad Y^{(3)}(m)=Y^{(4)}(m), \\
& \quad\left(V^{(3)}\right)_{z}(0)=0, \\
& \left.\begin{array}{c}
\left(V^{(4)}\right)_{z}=Y^{(4)} \\
\left(Y^{(4)}\right)_{z}=c Y^{(4)}-g\left(h_{-}\left(V^{(4)}\right), V^{(4)} ; \gamma\right)
\end{array}\right\} \quad z \in(m, \infty), \\
& V^{(4)}(\infty)=v_{-}(\gamma), \quad Y^{(4)}(\infty)=0, \\
& V^{(4)}(m)=\beta_{I}(-c ; \tau),
\end{aligned}
$$

and

$$
V^{(2)}(0)=V^{(3)}(0)
$$

If each problem (3.12a)-(3.12d) has a solution, we write it as $\left(V_{p}^{(i)}(z ; c ; \tau, \gamma), Y_{p}^{(i)}\right.$ $(z ; c ; \tau, \gamma))(i=1,2,3,4) . \quad \ell(m)$ is determined as a function of $c, \tau$, and $\gamma$, say, $\ell_{p}(c ; \tau, \gamma)\left(m_{p}(c ; \tau, \gamma)\right)$, by the time taken to traverse $\Gamma_{2}\left(\Gamma_{3}\right)$ from the point $A(C)$ to $B(D)$ (see Figure $9,(\mathrm{~b})$ and $(\mathrm{c})$ ).

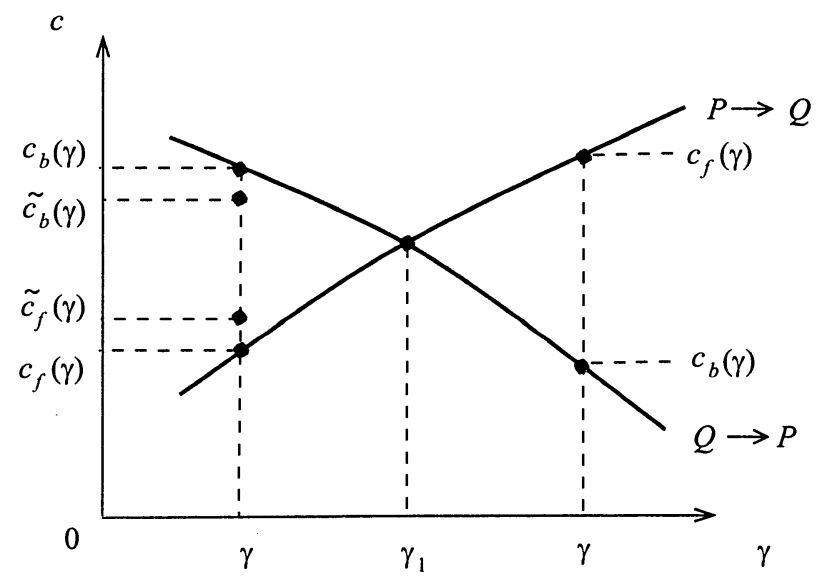

FIGURE 8. Coexistence of front and back waves.

First, we fix $\gamma$ arbitrarily satisfying $\gamma<\gamma_{1}$ and define $c_{f}(\gamma), c_{b}(\gamma)$ as in Figure 8 . $\left(v_{-}(\gamma), 0\right)$ and $\left(v_{+}(\gamma), 0\right)$ are critical points of $(3.12 \mathrm{a}),(3.12 \mathrm{~d})$ and $(3.12 \mathrm{~b}),(3.12 \mathrm{c})$, respectively. Using the theory of ordinary differential equations, we find that for $c=c_{b}(\gamma)$, there exist unique trajectories

$$
\Gamma_{i} \equiv\left(V_{p}^{(i)}\left(z ; c_{b}(\gamma) ; \tau, \gamma\right), Y_{p}^{(i)}\left(z ; c_{b}(\gamma) ; \tau, \gamma\right)\right)
$$

satisfying (3.12a)-(3.12d) (see Figure $9(\mathrm{a}))$. The trajectory $\Gamma_{1}$ emanates from the saddle point $O=\left(v_{-}(\gamma), 0\right)$ and reaches $A$. The two relations $\beta_{I}\left(c_{f}(\gamma) ; \tau\right)=\beta_{O}\left(c_{f}(\gamma) ; \gamma\right)$ and $\beta_{I}^{\prime}\left(c_{f}(\gamma) ; \tau\right)<\beta_{O}^{\prime}\left(c_{f}(\gamma) ; \gamma\right)$ imply that

$$
0=v_{-}(\gamma)<\beta_{I}\left(c_{b}(\gamma) ; \tau\right)<\beta_{O}\left(c_{b}(\gamma) ; \gamma\right)<v_{+}(\gamma)
$$

because of the relation $c_{f}(\gamma)<c_{b}(\gamma)$. By virtue of this, $\Gamma_{2}$ starts out at $A$ and reaches $B$. $\ell$ is determined by the time $\ell_{p}\left(c_{b}(\gamma) ; \tau, \gamma\right)$. On the other hand, $\Gamma_{3}$ emanates from the saddle point $\left(v_{+}(\gamma), 0\right)$ and reaches $D$. Because $\beta_{I}\left(-c_{b}(\gamma) ; \tau\right)=\beta_{O}\left(-c_{b}(\gamma) ; \gamma\right)$, 
$\Gamma_{4}$ starts out at $D$ and reaches the point $O$. In this case, we must take $m$ as $m_{p}\left(c_{b}(\gamma) ; \tau, \gamma\right)=\infty$. Let us choose $c=\tilde{c}_{b}(\gamma)\left(<c_{b}(\gamma)\right)$ close to $c_{b}(\gamma)$. By using the continuous dependence of the trajectories on $c$ and the relation $\beta_{I}\left(-\tilde{c}_{b}(\gamma) ; \tau\right)<$ $\beta_{O}\left(-\tilde{c}_{b}(\gamma) ; \gamma\right)$, we know that the trajectories in Figure $9(\mathrm{a})$ are slightly perturbed and changed into the ones in Figure $9(\mathrm{~b})$. For this $c, m$ is defined by the time $m_{p}\left(\tilde{c}_{b}(\gamma) ; \tau, \gamma\right)$ and remains finite. Here, define

$$
L(c ; \tau, \gamma) \equiv V^{(2)}(0 ; c ; \tau, \gamma)-V^{(3)}(0 ; c ; \tau, \gamma)
$$

We see that $L$ is a continuous function of $c$ and satisfies $L\left(\tilde{c}_{b}(\gamma) ; \tau, \gamma\right)<0 . L$ can be regarded as the distance with a sign from the singular stable manifold to the singular unstable manifold with respect to $O=\left(v_{-}(\gamma), 0\right)$ of the systems $(3.12 \mathrm{a})-(3.12 \mathrm{~d})$, which is closely related to a Melnikov function in [2] or a separation in [6].

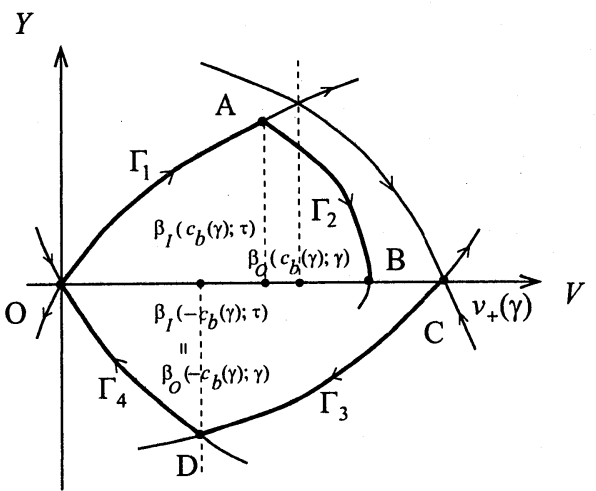

(a)

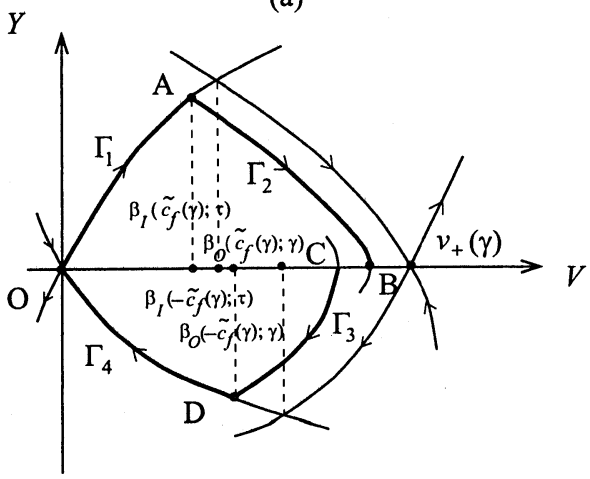

(c)

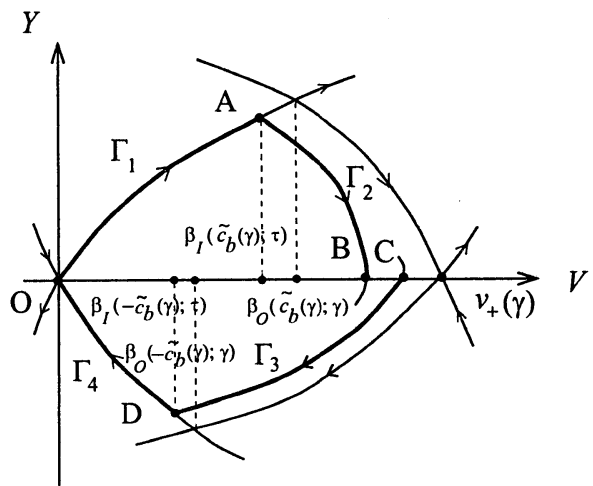

(b)

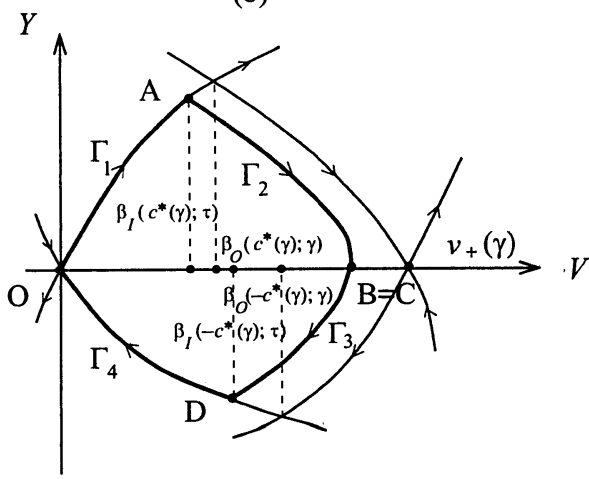

(d)

FiguRE 9. Trajectories of the singular limit problem for $\gamma<\gamma_{1}$ : (a) $c=c_{b}(\gamma),(\mathrm{b}) c=\tilde{c}_{b}(\gamma)<c_{b}(\gamma),(\mathrm{c}) c=\tilde{c}_{f}(\gamma)>c_{f}(\gamma),(\mathrm{d}) c=$ $c^{*}(\tau, \gamma)$.

Next, we fix $c=\tilde{c}_{f}(\gamma)\left(>c_{f}(\gamma)\right)$ close to $c_{f}(\gamma)$. Similar to the above analysis, there exist unique trajectories $\Gamma_{i}$ satisfying (3.12a)-(3.12d), as depicted in Figure 9(c). This implies that $L\left(\tilde{c}_{f}(\gamma) ; \tau, \gamma\right)>0$. Then, by the mean value theorem, we know that there exists $c_{p}^{+}(\tau, \gamma)\left(\tilde{c}_{f}(\gamma)<c_{p}^{+}(\tau, \gamma)<\tilde{c}_{b}(\gamma)\right)$ satisfying $L\left(c_{p}^{+}(\tau, \gamma) ; \tau, \gamma\right)=0$ (that is, $B=C)$, which indicates that we can get the solution trajectories $\Gamma_{i}$ satisfying 
(3.12a)-(3.12d) and (3.13). Here $\ell_{p}^{+}(\tau, \gamma)$ and $m_{p}^{+}(\tau, \gamma)$ are the time $\ell_{p}\left(c_{p}^{+}(\tau, \gamma) ; \tau, \gamma\right)$ and $m_{p}\left(c_{p}^{+}(\tau, \gamma) ; \tau, \gamma\right)$, respectively (see Figure $\left.9(\mathrm{~d})\right)$. We obtain the following theorem:

Theorem 3.1. For any $\gamma<\gamma_{1}$ in a neighborhood of $P_{1}$, there exist parameters $c_{p}^{+}(\tau, \gamma)$ in the interval $\left(c_{f}(\gamma), c_{b}(\gamma)\right), \ell_{p}^{+}(\tau, \gamma)$, and $m_{p}^{+}(\tau, \gamma)$ such that the singular limit problem (3.10a)-(3,10d), (3.11) has a solution

$$
V_{p}^{+}(z ; \tau, \gamma)= \begin{cases}V_{p}^{(1)}\left(z ; c_{p}^{+}(\tau, \gamma) ; \tau, \gamma\right) & z \in\left(-\infty,-\ell_{p}^{+}(\tau, \gamma)\right), \\ V_{p}^{(2)}\left(z ; c_{p}^{+}(\tau, \gamma) ; \tau, \gamma\right) & z \in\left(-\ell_{p}^{+}(\tau, \gamma), 0\right), \\ V_{p}^{(3)}\left(z ; c_{p}^{+}(\tau, \gamma) ; \tau, \gamma\right) & z \in\left(0, m_{p}^{+}(\tau, \gamma)\right), \\ V_{p}^{(4)}\left(z ; c_{p}^{+}(\tau, \gamma) ; \tau, \gamma\right) & z \in\left(m_{p}^{+}(\tau, \gamma), \infty\right) .\end{cases}
$$

$c_{p}^{+}(\tau, \gamma)$ is a singular limit velocity of a $P$-pulse wave and $\lim _{\gamma \uparrow \gamma_{1}} \ell_{p}^{+}(\tau, \gamma)=\infty=$ $\lim _{\gamma \uparrow \gamma_{1}} m_{p}^{+}(\tau, \gamma)$.

On the other hand, for any $\gamma>\gamma_{1}$, we cannot find solution trajectories $\Gamma_{i}$ satisfying (3.12a)-(3.12d) and (3.13) because for any $c$ in the interval $\left(c_{b}(\gamma), c_{f}(\gamma)\right)$, trajectories emanating from the point $O=\left(v_{-}(\gamma), 0\right)$ can never return to the same point (see Figure 10).

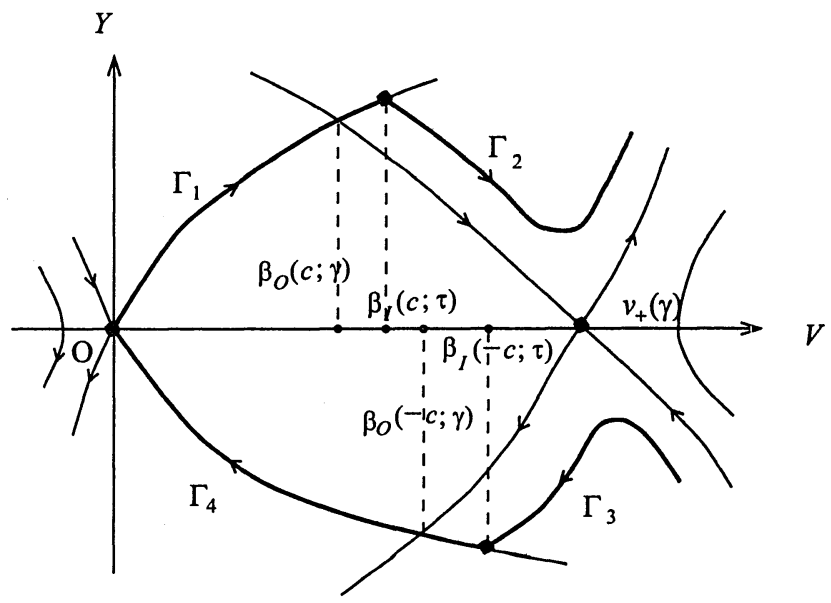

FIGURE 10. Trajectories of the singular limit problem for $\gamma>\gamma_{1}$.

For the singular limit problem of a $Q$-pulse wave:

$$
\begin{aligned}
& \left(V^{(1)}\right)_{z z}-c\left(V^{(1)}\right)_{z}+g\left(h_{+}\left(V^{(1)}\right), V^{(1)} ; \gamma\right)=0 \quad z \in(-\infty,-\ell) \\
& V^{(1)}(-\infty)=v_{+}(\gamma), \quad V^{(1)}(-\ell)=\beta_{I}(-c ; \tau), \\
& \left(V^{(2)}\right)_{z z}-c\left(V^{(2)}\right)_{z}+g\left(h_{-}\left(V^{(2)}\right), V^{(2)} ; \gamma\right)=0 \quad z \in(-\ell, 0) \\
& V^{(2)}(-\ell)=\beta_{I}(-c ; \tau), \quad\left(V^{(2)}\right)_{z}(-\ell)=\left(V^{(1)}\right)_{z}(-\ell) \\
& \left(V^{(2)}\right)_{z}(0)=0, \\
& \left(V^{(3)}\right)_{z z}-c\left(V^{(3)}\right)_{z}+g\left(h_{-}\left(V^{(3)}\right), V^{(3)} ; \gamma\right)=0 \quad z \in(0, m) \\
& \left(V^{(3)}\right)_{z}(0)=0, \\
& V^{(3)}(m)=\beta_{I}(c ; \tau), \quad\left(V^{(3)}\right)_{z}(m)=\left(V^{(4)}\right)_{z}(m)
\end{aligned}
$$




$$
\begin{aligned}
& \left(V^{(4)}\right)_{z z}-c\left(V^{(4)}\right)_{z}+g\left(h_{+}\left(V^{(4)}\right), V^{(4)} ; \gamma\right)=0 \quad z \in(m, \infty) \\
& V^{(4)}(m)=\beta_{I}(c ; \tau), \quad V^{(4)}(\infty)=v_{+}(\gamma)
\end{aligned}
$$

and

$$
\left(V^{(2)}\right)(0)=\left(V^{(3)}\right)(0),
$$

let $V_{q}^{(i)}(z ; c ; \tau, \gamma)$ be solutions of $(3.15 \mathrm{a})-(3.15 \mathrm{~d}) . \ell(m)$ is determined as a function of $c, \tau$, and $\gamma$, say $\ell_{q}(c ; \tau, \gamma)\left(m_{q}(c ; \tau, \gamma)\right)$, satisfying (3.15b) ((3.15c)). We can show the following results by using a method similar to the above:

Theorem 3.2. For any $\gamma>\gamma_{1}$ in a neighborhood of $P_{1}$, there exists a parameter $c_{q}^{+}(\tau, \gamma)$ in the interval $\left(c_{b}(\gamma), c_{f}(\gamma)\right)$ such that the singular limit problem (3.15a)(3.15d), (3.16) has a solution

$$
V_{q}^{+}(z ; \tau, \gamma)= \begin{cases}V_{q}^{(1)}\left(z ; c_{q}^{+}(\tau, \gamma) ; \tau, \gamma\right) & z \in\left(-\infty,-\ell_{q}^{+}(\tau, \gamma)\right), \\ V_{q}^{(2)}\left(z ; c_{q}^{+}(\tau, \gamma) ; \tau, \gamma\right) & z \in\left(-\ell_{q}^{+}(\tau, \gamma), 0\right), \\ V_{q}^{(3)}\left(z ; c_{q}^{+}(\tau, \gamma) ; \tau, \gamma\right) & z \in\left(0, m_{q}^{+}(\tau, \gamma)\right), \\ V_{q}^{(4)}\left(z ; c_{q}^{+}(\tau, \gamma) ; \tau, \gamma\right) & z \in\left(m_{q}^{+}(\tau, \gamma), \infty\right),\end{cases}
$$

where $\ell_{q}^{+}(\tau, \gamma)=\ell_{q}\left(c_{q}^{+}(\tau, \gamma) ; \tau, \gamma\right)$ and $m_{q}^{+}(\tau, \gamma)=m_{q}\left(c_{q}^{+}(\tau, \gamma) ; \tau, \gamma\right) . c_{q}^{+}(\tau, \gamma)$ is a singular limit velocity of a $Q$-pulse wave and $\lim _{\gamma \downarrow \gamma_{1}} \ell_{q}^{+}(\tau, \gamma)=\infty=\lim _{\gamma \downarrow \gamma_{1}} m_{q}^{+}(\tau, \gamma)$.

If we get a singular limit $P$-pulse $\left(Q\right.$-pulse) wave $V_{p}^{+}(z ; \tau, \gamma)\left(V_{q}^{+}(z ; \tau, \gamma)\right)$ with a velocity $c_{p}^{+}(\tau, \gamma)\left(c_{q}^{+}(\tau, \gamma)\right)$, then $V_{p}^{+}(-z ; \tau, \gamma)\left(V_{q}^{+}(-z ; \tau, \gamma)\right)$ is a singular limit $P$ pulse $\left(Q\right.$-pulse) wave with a velocity $-c_{p}(\tau, \gamma)\left(-c_{q}(\tau, \gamma)\right)$. Then, in a neighborhood of the point $P_{3}$, we have

Corollary 3.1. For any $\gamma<\gamma_{1}\left(\gamma>\gamma_{1}\right)$ in a neighborhood of $P_{3}$, there exists a singular limit P-pulse (Q-pulse) wave $V_{p}^{-}(z ; \tau, \gamma)=V_{p}^{+}(-z ; \tau, \gamma)\left(V_{q}^{-}(z ; \tau, \gamma)=\right.$ $\left.V_{q}^{+}(-z ; \tau, \gamma)\right)$ with a singular limit velocity $c_{p}^{-}(\tau, \gamma)=-c_{p}^{+}(\tau, \gamma)\left(c_{q}^{-}(\tau, \gamma)=-c_{q}^{+}(\tau, \gamma)\right)$.

Also, in neighborhoods of $P_{2}, P_{4}$, or $P_{5}$, results similar to the case of $P_{1}$ or $P_{3}$ hold.

Theorem 3.3. For any $\gamma<\gamma_{1}\left(\gamma>\gamma_{1}\right)$ in a neighborhood of $P_{i}(i=2,4,5)$, there exists a singular limit $P$-pulse $\left(Q\right.$-pulse) wave $V_{p}^{0}(z ; \tau, \gamma)\left(V_{q}^{0}(z ; \tau, \gamma)\right)$ with a singular limit velocity $c_{p}^{0}(\tau, \gamma)\left(c_{q}^{0}(\tau, \gamma)\right)$.

The above analysis of the singular limit problem of $P$-pulse or $Q$-pulse waves shows that in a neighborhood of each point $P_{i}(i=1,2,3,4,5)$, there exists a singular limit $P$-pulse wave for $\gamma<\gamma_{1}$ and a singular limit $Q$-pulse wave for $\gamma>\gamma_{1}$. To prove the existence of an exact $P$-pulse or $Q$-pulse wave solutions of the original problems (3.1), (3.2) or (3.1), (3.3), as we have seen in $\S 2$, we must show the transversality condition

$$
\frac{\partial}{\partial c} L(c ; \tau, \gamma) \neq 0
$$

at each singular limit velocity $c$ (see [4]). Of course, at the point $P_{4}$, standing front and back waves with zero velocity coexist. But both waves do not satisfy the transversality condition (see Figure 5(b)). So in a neighborhood of $P_{4}$, we cannot show the transversality condition for $P$-pulse or $Q$-pulse waves with the aid of these standing front and back waves (see $\S 4$ ). In order to overcome these difficulties, a more careful and delicate analysis will be required. At other points, say, $P_{1}, P_{2}, P_{3}$, and $P_{5}$, we can show the transversality condition. For the proof of the existence of an exact pulse wave, it suffices to show the transversality condition (3.17). But for the stability 
property, it is important to know the sign of the left-hand side of (3.17), which corresponds to the crossing behavior of the stable and unstable manifolds with respect to the equilibrium points for the singular limit problem. We will state this in the next section. Therefore, we conclude with the following results for local bifurcation diagrams of traveling $P$-pulse or $Q$-pulse waves in the $(c, \gamma)$-parameter space near the points $P_{1}, P_{2}, P_{3}$ and $P_{5}$ (see Figure 11).

Theorem 3.4. (1) In a neighborhood of $P_{1}\left(P_{3}\right)$, a singular limit velocity $c=c_{p}^{+}(\tau, \gamma)$ $>0\left(c=c_{p}^{-}(\tau, \gamma)<0\right)\left(\gamma<\gamma_{1}\right)$ of a $P$-pulse wave bifurcates tangentially from the curve $P \rightarrow Q(Q \rightarrow P)$ at $\gamma=\gamma_{1}$ and a singular limit velocity $c=c_{q}^{+}(\tau, \gamma)>0$ $\left(c=c_{q}^{-}(\tau, \gamma)<0\right)\left(\gamma>\gamma_{1}\right)$ of a $Q$-pulse wave bifurcates tangentially from the curve $Q \rightarrow P(P \rightarrow Q)$ at $\gamma=\gamma_{1}$.

(2) In a neighborhood of $P_{2}$ or $P_{5}$, a singular limit velocity $c=c_{p}^{0}(\tau, \gamma)\left(\gamma<\gamma_{1}\right)$ of a P-pulse wave bifurcates from the curve $P \rightarrow Q$ and $Q \rightarrow P$ at $\gamma=\gamma_{1}$, which satisfies $\frac{\partial}{\partial \gamma} c_{p}^{0}\left(\tau, \gamma_{1}\right)=0$ and a singular limit velocity $c=c_{q}^{0}(\tau, \gamma)\left(\gamma>\gamma_{1}\right)$ of a $Q$ pulse wave bifurcates from the curve $P \rightarrow Q$ and $Q \rightarrow P$ at $\gamma=\gamma_{1}$, which satisfies $\frac{\partial}{\partial \gamma} c_{q}^{0}\left(\tau, \gamma_{1}\right)=0$.

$c$

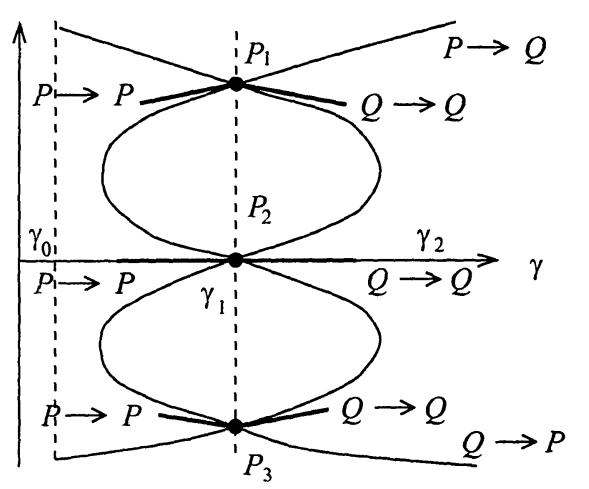

(a)

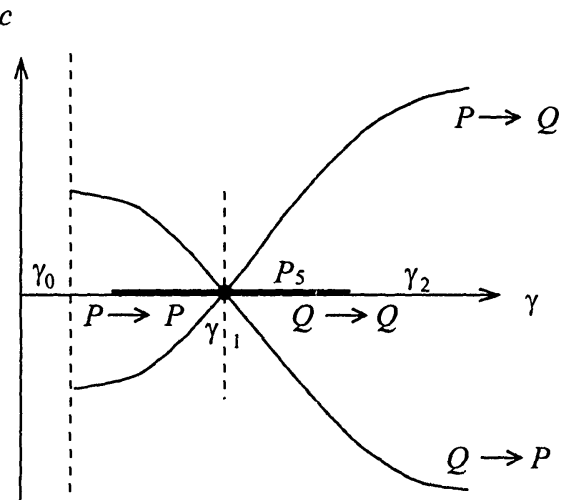

(b)

FIGURE 11. Bifurcation of pulse waves from front and back waves:

(a) $0<\tau<\tau_{c}\left(\gamma_{1}\right)$, (b) $\tau>\tau_{c}\left(\gamma_{1}\right)$.

For the proof, we must show the transversality condition (3.17) and compute the value of $\frac{\partial}{\partial \gamma} c\left(\tau, \gamma_{1}\right)$ of each singular limit velosity $c(\tau, \gamma)$, which will be done in the next section.

We note that this theorem is obtained in [6] by using homoclinic bifurcation theory. We already know the existence of standing $P$-pulse waves for $\gamma<\gamma_{1}$ and $Q$-pulse waves for $\gamma>\gamma_{1}$. Indeed, these are equal to $c=c_{p}^{0}(\tau, \gamma)\left(\gamma<\gamma_{1}\right)$ and $c=c_{q}^{0}(\tau, \gamma)\left(\gamma>\gamma_{1}\right)$ in a neighborhood of $P_{2}$ or $P_{5}$ of the $(c, \gamma)$-parameter space.

Remark 3.1. In a neighborhood of $P_{2}$ or $P_{5}, c_{p}^{0}(\tau, \gamma)=0$ for $\gamma<\gamma_{1}$ and $c_{q}^{0}(\tau, \gamma)=0$ for $\gamma>\gamma_{1}$.

\section{Crossing behavior for singular limit solutions}

In this section, we restrict ourselves to the case $\gamma<\gamma_{1}$. The case $\gamma>\gamma_{1}$ can be treated similarly. We use the notation $c^{*}\left(=c^{*}(\tau, \gamma)\right)$ in place of $c_{p}^{+}(\tau, \gamma), c_{p}^{-}(\tau, \gamma)$ 
or $c_{p}^{0}(\tau, \gamma)$ for simplicity. $\ell^{*}$ and $m^{*}$ are the values determined by the singular limit problem of a $P$-pulse wave with $c=c^{*}$. We establish the crossing behavior of the stable and unstable manifolds corresponding to each singular limit solution, which plays an essential role in proving the existence of an exact solution and its stability property [4]. Furthermore, we compute the slope of the bifurcated singular limit velocities of singular limit pulse waves at $\gamma=\gamma_{1}$ (see Theorem 3.4). For this purpose, we have to examine the dependence of the parameters $c, \ell, m$, and $\gamma$ on a singular limit pulse wave. First, let us consider the problems:

$$
\begin{aligned}
& \left(V^{(1)}\right)_{z z}-c\left(V^{(1)}\right)_{z}+g\left(h_{-}\left(V^{(1)}\right), V^{(1)} ; \gamma\right)=0 \quad z \in(-\infty,-\ell), \\
& V^{(1)}(-\infty)=v_{-}(\gamma), \quad V^{(1)}(-\ell)=\beta_{I}(c ; \tau), \\
& \left(V^{(2)}\right)_{z z}-c\left(V^{(2)}\right)_{z}+g\left(h_{+}\left(V^{(2)}\right), V^{(2)} ; \gamma\right)=0 \quad z \in(-\ell, 0), \\
& V^{(2)}(-\ell)=\beta_{I}(c ; \tau), \quad\left(V^{(2)}\right)_{z}(0)=0, \\
& \left(V^{(3)}\right)_{z z}-c\left(V^{(3)}\right)_{z}+g\left(h_{+}\left(V^{(3)}\right), V^{(3)} ; \gamma\right)=0 \quad z \in(0, m), \\
& \left(V^{(3)}\right)_{z}(0)=0, \quad V^{(3)}(m)=\beta_{I}(-c ; \tau), \\
& \left(V^{(4)}\right)_{z z}-c\left(V^{(4)}\right)_{z}+g\left(h_{-}\left(V^{(4)}\right), V^{(4)} ; \gamma\right)=0 \quad z \in(m, \infty), \\
& V^{(4)}(m)=\beta_{I}(-c ; \tau), \quad V^{(4)}(\infty)=v_{+}(\gamma) \text {. }
\end{aligned}
$$

It is easy to find solutions of (4.1a)-(4.1d) by using the phase plane analysis. Then, we write the solutions of (4.1a)-(4.1d) as $V^{(1)}(z ; c, \ell ; \gamma), V^{(2)}(z ; c, \ell ; \gamma), V^{(3)}(z ; c, m ; \gamma)$ and $V^{(4)}(z ; c, m ; \gamma)$, respectively. Here we do not write the dependence of $\tau$ explicitly because it is not so important for our analysis in this section. For these solutions, we have the next lemma.

Lemma 4.1. For any $(c, \ell, m, \gamma)$ in some neighborhood of $\left(c^{*}, \ell^{*}, m^{*}, \gamma_{1}\right)$, we have the following relations:

$$
\begin{gathered}
\frac{\partial}{\partial c}\left(V^{(1)}\right)_{z}(-\ell ; c, \ell ; \gamma)=\frac{\beta_{I}^{\prime}(c)\left(V^{(1)}\right)_{z z}(-\ell)}{\left(V^{(1)}\right)_{z}(-\ell)}+\int_{-\infty}^{-\ell} \frac{e^{-c(z+\ell)}\left(\left(V^{(1)}\right)_{z}(z)\right)^{2} d z}{\left(V^{(1)}\right)_{z}(-\ell)}, \\
\frac{\partial}{\partial \ell}\left(V^{(1)}\right)_{z}(-\ell ; c, \ell ; \gamma)=\left(V^{(1)}\right)_{z z}(-\ell), \\
\frac{\partial}{\partial \gamma}\left(V^{(1)}\right)_{z}(-\ell ; c, \ell ; \gamma)=\int_{-\infty}^{-\ell} e^{-c(z+\ell)}\left(V^{(1)}\right)_{z}(z) V^{(1)}(z) d z /\left(V^{(1)}\right)_{z}(-\ell), \\
\frac{\partial}{\partial c} V^{(2)}(0 ; c, \ell ; \gamma)=\frac{1}{A_{1}^{\prime}(0 ; c, \ell ; \gamma)}\left\{\frac{\beta_{I}^{\prime}(c)}{\left(V^{(2)}\right)_{z}(-\ell)}-\int_{-\ell}^{0} e^{-c z} A_{1}(z ; c, \ell ; \gamma)\left(V^{(2)}\right)_{z}(z) d z\right\}
\end{gathered}
$$




$$
\begin{aligned}
& \frac{\partial}{\partial c}\left(V^{(2)}\right)_{z}(-\ell ; c, \ell ; \gamma)= \\
& \frac{\beta_{I}^{\prime}(c)}{\left(V^{(2)}\right)_{z}(-\ell)}\left\{\left(V^{(2)}\right)_{z z}(-\ell)-\frac{\left(V^{(2)}\right)_{z z}(0)}{A_{1}^{\prime}(0 ; c, \ell ; \gamma)} \cdot \frac{e^{-c \ell}}{\left(V^{(2)}\right)_{z}(-\ell)}\right\} \\
& -\frac{e^{-c \ell}}{\left(V^{(2)}\right)_{z}(-\ell)}\left\{\int_{-\ell}^{0} e^{-c z}\left(\left(V^{(2)}\right)_{z}(z)\right)^{2} d z\right. \\
& \left.-\frac{\left(V^{(2)}\right)_{z z}(0)}{A_{1}^{\prime}(0 ; c, \ell ; \gamma)} \int_{-\ell}^{0} e^{-c z} A_{1}(z ; c, \ell ; \gamma)\left(V^{(2)}\right)_{z}(z) d z\right\} \\
& \frac{\partial}{\partial \ell} V^{(2)}(0 ; c, \ell ; \gamma)=\frac{1}{A_{1}^{\prime}(0 ; c, \ell ; \gamma)} \\
& \frac{\partial}{\partial \ell}\left(V^{(2)}\right)_{z}(-\ell ; c, \ell ; \gamma)=\left(V^{(2)}\right)_{z z}(-\ell)-\frac{\left(V^{(2)}\right)_{z z}(0)}{A_{1}^{\prime}(0 ; c, \ell ; \gamma)} \cdot \frac{e^{-c \ell}}{\left(V^{(2)}\right)_{z}(-\ell)} \\
& \frac{\partial}{\partial \gamma} V^{(2)}(0 ; c, \ell ; \gamma)=-\frac{1}{A_{1}^{\prime}(0 ; c, \ell ; \gamma)} \int_{-\ell}^{0} e^{-c z} A_{1}(z ; c, \ell ; \gamma) V^{(2)}(z) d z \\
& \frac{\partial}{\partial \gamma}\left(V^{(2)}\right)_{z}(-\ell ; c, \ell ; \gamma)=-\frac{e^{-c \ell}}{\left(V^{(2)}\right)_{z}(-\ell)}\left\{\int_{-\ell}^{0} e^{-c z}\left(V^{(2)}\right)_{z}(z) V^{(2)}(z) d z\right. \\
& \left.+\frac{\left(V^{(2)}\right)_{z z}(0)}{A_{1}^{\prime}(0 ; c, \ell ; \gamma)} \int_{-\ell}^{0} e^{-c z} A_{1}(z ; c, \ell ; \gamma) V^{(2)}(z) d z\right\} \\
& \frac{\partial}{\partial c} V^{(3)}(0 ; c, m ; \gamma)=\frac{1}{A_{2}^{\prime}(0 ; c, m ; \gamma)}\left\{\frac{\beta_{I}^{\prime}(-c)}{\left(V^{(3)}\right)_{z}(m)}\right. \\
& \left.+\int_{0}^{m} e^{-c z} A_{2}(z ; c, m ; \gamma)\left(V^{(3)}\right)_{z}(z) d z\right\} \\
& \frac{\partial}{\partial c}\left(V^{(3)}\right)_{z}(m ; c, m ; \gamma)= \\
& -\frac{\beta_{I}^{\prime}(-c)}{\left(V^{(3)}\right)_{z}(m)}\left\{\left(V^{(3)}\right)_{z z}(m)+\frac{\left(V^{(3)}\right)_{z z}(0)}{A_{2}^{\prime}(0 ; c, m ; \gamma)} \cdot \frac{e^{c m}}{\left(V^{(3)}\right)_{z}(m)}\right\} \\
& +\frac{e^{c m}}{\left(V^{(3)}\right)_{z}(m)}\left\{\int_{0}^{m} e^{-c z}\left(\left(V^{(3)}\right)_{z}(z)\right)^{2} d z\right. \\
& \left.-\frac{\left(V^{(3)}\right)_{z z}(0)}{A_{2}^{\prime}(0 ; c, m ; \gamma)} \int_{0}^{m} e^{-c z} A_{2}(z ; c, m ; \gamma)\left(V^{(3)}\right)_{z}(z) d z\right\} \\
& \frac{\partial}{\partial m} V^{(3)}(0 ; c, m ; \gamma)=\frac{1}{A_{2}^{\prime}(0 ; c, m ; \gamma)} \\
& \frac{\partial}{\partial m}\left(V^{(3)}\right)_{z}(m ; c, m ; \gamma)=-\left(V^{(3)}\right)_{z z}(m)-\frac{\left(V^{(3)}\right)_{z z}(0)}{A_{2}^{\prime}(0 ; c, m ; \gamma)} \cdot \frac{e^{c m}}{\left(V^{(3)}\right)_{z}(m)} \\
& \frac{\partial}{\partial \gamma} V^{(3)}(0 ; c, m ; \gamma)=\frac{1}{A_{2}^{\prime}(0 ; c, m ; \gamma)} \int_{0}^{m} e^{-c z} A_{2}(z ; c, m ; \gamma) V^{(3)}(z) d z
\end{aligned}
$$




$$
\begin{gathered}
\frac{\partial}{\partial \gamma}\left(V^{(3)}\right)_{z}(m ; c, m ; \gamma)=\frac{e^{c m}}{\left(V^{(3)}\right)_{z}(m)}\left\{\int_{0}^{m} e^{-c z}\left(V^{(3)}\right)_{z}(z) V^{(3)}(z) d z\right. \\
\left.+\frac{\left(V^{(3)}\right)_{z z}(0)}{A_{2}^{\prime}(0 ; c, m ; \gamma)} \int_{0}^{m} e^{-c z} A_{2}(z ; c, m ; \gamma) V^{(3)}(z) d z\right\} \\
\frac{\partial}{\partial c}\left(V^{(4)}\right)_{z}(m ; c, m ; \gamma)=-\frac{\beta_{I}^{\prime}(-c)\left(V^{(4)}\right)_{z z}(m)}{\left(V^{(4)}\right)_{z}(m)} \\
\frac{\partial}{\partial m}\left(V^{(4)}\right)_{z}(m ; c, m ; \gamma)=-\left(V^{(4)}\right)_{z z}(m) \\
\frac{\partial}{\partial \gamma}\left(V^{(4)}\right)_{z}(m ; c, m ; \gamma)=-\int_{m}^{\infty(z-m)}\left(\left(V^{(4)}\right)_{z}(z)\right)^{2} d z \\
\end{gathered}
$$

where

$$
\begin{aligned}
& A_{1}(z ; c, \ell ; \gamma)=\left(V^{(2)}\right)_{z}(z) \int_{-\ell}^{z} e^{c y}\left(\left(V^{(2)}\right)_{y}(y)\right)^{-2} d y \\
& A_{2}(z ; c, m ; \gamma)=\left(V^{(3)}\right)_{z}(z) \int_{z}^{m} e^{c y}\left(\left(V^{(3)}\right)_{y}(y)\right)^{-2} d y
\end{aligned}
$$

and $A_{1}^{\prime}=\frac{d}{d z} A_{1}, A_{2}^{\prime}=\frac{d}{d z} A_{2}$. Moreover, we used the relation $g(u, v ; \gamma)=u-\gamma v$.

The proof will be given in the Appendix.

Put

$$
\begin{aligned}
P(c, \ell ; \gamma) & \equiv\left(V^{(1)}\right)_{z}(-\ell ; c, \ell ; \gamma)-\left(V^{(2)}\right)_{z}(-\ell ; c, \ell ; \gamma) \\
Q(c, \ell, m ; \gamma) & \equiv\left(V^{(2)}\right)(0 ; c, \ell ; \gamma)-\left(V^{(3)}\right)(0 ; c, m ; \gamma), \\
R(c, m ; \gamma) & \equiv\left(V^{(3)}\right)_{z}(m ; c, m ; \gamma)-\left(V^{(4)}\right)_{z}(m ; c, m ; \gamma)
\end{aligned}
$$

From the relations (4.3), (4.8), (4.14), and (4.18), it follows that

$$
\begin{aligned}
\frac{\partial}{\partial \ell} P(c, \ell ; \gamma)=- & \left(V^{(1)}\right)_{z z}(-\ell ; c, \ell ; \gamma)+\frac{\partial}{\partial \ell}\left(V^{(1)}\right)_{z}(-\ell ; c, \ell ; \gamma) \\
& +\left(V^{(2)}\right)_{z z}(-\ell ; c, \ell ; \gamma)-\frac{\partial}{\partial \ell}\left(V^{(2)}\right)_{z}(-\ell ; c, \ell ; \gamma) \\
= & \frac{\left(V^{(2)}\right)_{z z}(0)}{A_{1}^{\prime}(0 ; c, \ell ; \gamma)} \cdot \frac{e^{-c \ell}}{\left(V^{(2)}\right)_{z}(-\ell)}<0, \\
\frac{\partial}{\partial m} R(c, m ; \gamma)= & \left(V^{(3)}\right)_{z z}(m ; c, m ; \gamma)+\frac{\partial}{\partial m}\left(V^{(3)}\right)_{z}(m ; c, m ; \gamma) \\
& -\left(V^{(4)}\right)_{z z}(m ; c, m ; \gamma)-\frac{\partial}{\partial m}\left(V^{(4)}\right)_{z}(m ; c, m ; \gamma) \\
= & -\frac{\left(V^{(3)}\right)_{z z}(0)}{A_{2}^{\prime}(0 ; c, m ; \gamma)} \cdot \frac{e^{c m}}{\left(V^{(3)}\right)_{z}(m)}<0 .
\end{aligned}
$$


Then $\ell=\ell(c ; \gamma)$ and $m=m(c ; \gamma)$ are uniquely determined such that $P(c, \ell(c ; \gamma) ; \gamma)=$ 0 and $R(c, m(c ; \gamma) ; \gamma)=0$, respectively. Furthermore, by Lemma 4.1, we find that

$$
\begin{aligned}
& \frac{\partial}{\partial c} \ell(c ; \gamma)=-\left.\frac{\frac{\partial}{\partial c} P(c, \ell ; \gamma)}{\frac{\partial}{\partial \ell} P(c, \ell ; \gamma)}\right|_{\ell=\ell(c ; \gamma)}, \\
& \frac{\partial}{\partial \gamma} \ell(c ; \gamma)=-\left.\frac{\frac{\partial}{\partial \gamma} P(c, \ell ; \gamma)}{\frac{\partial}{\partial \ell} P(c, \ell ; \gamma)}\right|_{\ell=\ell(c ; \gamma)}, \\
& \frac{\partial}{\partial c} m(c ; \gamma)=-\left.\frac{\frac{\partial}{\partial c} R(c, m ; \gamma)}{\frac{\partial}{\partial m} R(c, m ; \gamma)}\right|_{m=m(c ; \gamma)}, \\
& \frac{\partial}{\partial \gamma} m(c ; \gamma)=-\left.\frac{\frac{\partial}{\partial \gamma} R(c, m ; \gamma)}{\frac{\partial}{\partial m} P(c, m ; \gamma)}\right|_{m=m(c ; \gamma)},
\end{aligned}
$$

where

$$
\begin{aligned}
& \frac{\partial}{\partial c} P(c, \ell ; \gamma)=\beta_{I}^{\prime}(c)\left\{\frac{\left(V^{(1)}\right)_{z z}(-\ell)}{\left(V^{(1)}\right)_{z}(-\ell)}-\frac{\left(V^{(2)}\right)_{z z}(-\ell)}{\left(V^{(2)}\right)_{z}(-\ell)}\right\} \\
& +\frac{\int_{-\infty}^{-\ell} e^{-c(z+\ell)}\left(\left(V^{(1)}\right)_{z}(z)\right)^{2} d z}{\left(V^{(1)}\right)_{z}(-\ell)}+\frac{\int_{-\ell}^{0} e^{-c(z+\ell)}\left(\left(V^{(2)}\right)_{z}(z)\right)^{2} d z}{\left(V^{(2)}\right)_{z}(-\ell)} \\
& +\frac{\left(V^{(2)}\right)_{z z}(0)}{A_{1}^{\prime}(0 ; c, \ell ; \gamma)} \cdot \frac{e^{-c \ell}}{\left(V^{(2)}\right)_{z}(-\ell)}\left\{\frac{\beta_{I}^{\prime}(c)}{\left(V^{(2)}\right)_{z}(-\ell)}\right. \\
& \left.-\int_{-\ell}^{0} e^{-c z} A_{1}(z ; c, \ell ; \gamma)\left(V^{(2)}\right)_{z}(z) d z\right\} \\
& \frac{\partial}{\partial \gamma} P(c, \ell ; \gamma)=\frac{\int_{-\infty}^{-\ell} e^{-c(z+\ell)}\left(V^{(1)}\right)_{z}(z) V^{(1)}(z) d z}{\left(V^{(1)}\right)_{z}(-\ell)}+\frac{\int_{-\ell}^{0} e^{-c(z+\ell)}\left(V^{(2)}\right)_{z}(z) V^{(2)}(z) d z}{\left(V^{(2)}\right)_{z}(-\ell)} \\
& -\frac{\left(V^{(2)}\right)_{z z}(0)}{A_{1}^{\prime}(0 ; c, \ell ; \gamma)} \cdot \frac{1}{\left(V^{(2)}\right)_{z}(-\ell)} \int_{-\ell}^{0} e^{-c(z+\ell)} A_{1}(z ; c, \ell ; \gamma) V^{(2)}(z) d z \\
& \frac{\partial}{\partial c} R(c, m ; \gamma)=-\beta_{I}^{\prime}(-c)\left\{\frac{\left(V^{(3)}\right)_{z z}(m)}{\left(V^{(3)}\right)_{z}(m)}-\frac{\left(V^{(4)}\right)_{z z}(m)}{\left(V^{(4)}\right)_{z}(m)}\right\} \\
& +\frac{\int_{0}^{m} e^{-c(z-m)}\left(\left(V^{(3)}\right)_{z}(z)\right)^{2} d z}{\left(V^{(3)}\right)_{z}(m)}+\frac{\int_{m}^{\infty} e^{-c(z-m)}\left(\left(V^{(4)}\right)_{z}(z)\right)^{2} d z}{\left(V^{(4)}\right)_{z}(m)} \\
& +\frac{\left(V^{(3)}\right)_{z z}(0)}{A_{2}^{\prime}(0 ; c, m ; \gamma)} \cdot \frac{e^{c m}}{\left(V^{(3)}\right)_{z}(m)}\left\{-\frac{\beta_{I}^{\prime}(-c)}{\left(V^{(3)}\right)_{z}(m)}\right. \\
& \left.-\int_{0}^{m} e^{-c z} A_{2}(z ; c, m ; \gamma)\left(V^{(3)}\right)_{z}(z) d z\right\} \\
& \frac{\partial}{\partial \gamma} R(c, m ; \gamma)=\frac{\int_{0}^{m} e^{-c(z-m)}\left(V^{(3)}\right)_{z}(z) V^{(3)}(z) d z}{\left(V^{(3)}\right)_{z}(m)}+\frac{\int_{m}^{\infty} e^{-c(z-m)}\left(V^{(4)}\right)_{z}(z) V^{(4)}(z) d z}{\left(V^{(4)}\right)_{z}(m)} \\
& -\frac{\left(V^{(3)}\right)_{z z}(0)}{A_{2}^{\prime}(0 ; c, m ; \gamma)} \cdot \frac{1}{\left(V^{(3)}\right)_{z}(m)} \int_{0}^{m} e^{-c(z-m)} A_{2}(z ; c, m ; \gamma) V^{(3)}(z) d z .
\end{aligned}
$$

Finally, let us define $c=c(\gamma)$ satisfying

$$
Q(c, \ell(c ; \gamma), m(c ; \gamma) ; \gamma)=0
$$


Note that the left-hand side of (4.21) is equal to $L(c ; \gamma)$ defined in (3.14). We know that $c(\gamma)=c^{*}$. Our purpose is to evaluate

$$
\frac{\partial}{\partial c} L\left(c^{*} ; \gamma\right)=\frac{\partial Q}{\partial c}+\frac{\partial \ell}{\partial c} \cdot \frac{\partial Q}{\partial \ell}+\left.\frac{\partial m}{\partial c} \cdot \frac{\partial Q}{\partial m}\right|_{c=c^{*}, \ell=\ell^{*}, m=m^{*}}
$$

and

$$
\frac{d}{d \gamma} c^{*}(\gamma)=-\left.\frac{\frac{\partial}{\partial \gamma} Q+\frac{\partial}{\partial \gamma} \ell \cdot \frac{\partial}{\partial \ell} Q+\frac{\partial}{\partial \gamma} m \cdot \frac{\partial}{\partial m} Q}{\frac{\partial}{\partial c} Q+\frac{\partial}{\partial c} \ell \cdot \frac{\partial}{\partial \ell} Q+\frac{\partial}{\partial c} m \cdot \frac{\partial}{\partial m} Q}\right|_{c=c^{*}, \ell=\ell^{*}, m=m^{*}}
$$

Components of the above expressions are evaluated from Lemma 4.1 as follows:

$$
\begin{aligned}
\frac{\partial}{\partial c} Q(c, \ell, m ; \gamma) & =\frac{1}{A_{1}^{\prime}(0 ; c, \ell ; \gamma)}\left\{\frac{\beta_{I}^{\prime}(c)}{\left(V^{(2)}\right)_{z}(-\ell)}-\int_{-\ell}^{0} e^{-c z} A_{1}(z ; c, \ell ; \gamma)\left(V^{(2)}\right)_{z}(z) d z\right\} \\
+ & \frac{1}{A_{2}^{\prime}(0 ; c, m ; \gamma)}\left\{-\frac{\beta_{I}^{\prime}(-c)}{\left(V^{(3)}\right)_{z}(m)}-\int_{0}^{m} e^{-c z} A_{2}(z ; c, m ; \gamma)\left(V^{(3)}\right)_{z}(z) d z\right\} \\
\frac{\partial}{\partial \ell} Q(c, \ell, m ; \gamma)= & \frac{1}{A_{1}^{\prime}(0 ; c, \ell ; \gamma)}, \quad \frac{\partial}{\partial m} Q(c, \ell, m ; \gamma)=-\frac{1}{A_{2}^{\prime}(0 ; c, m ; \gamma)} \\
\frac{\partial}{\partial \gamma} Q(c, \ell, m ; \gamma)= & -\frac{1}{A_{1}^{\prime}(0 ; c, \ell ; \gamma)} \int_{-\ell}^{0} e^{-c z} A_{1}(z ; c, \ell ; \gamma) V^{(2)}(z) d z \\
& \quad-\frac{1}{A_{2}^{\prime}(0 ; c, m ; \gamma)} \int_{0}^{m} e^{-c z} A_{2}(z ; c, m ; \gamma) V^{(3)}(z) d z
\end{aligned}
$$

Thus, we have

$$
\begin{aligned}
& \frac{\partial}{\partial c} L\left(c^{*} ; \gamma\right)=\frac{\partial Q}{\partial c}+\frac{\partial \ell}{\partial c} \cdot \frac{\partial Q}{\partial \ell}+\left.\frac{\partial m}{\partial c} \cdot \frac{\partial Q}{\partial m}\right|_{c=c^{*}, \ell=\ell^{*}, m=m^{*}} \\
& =\frac{1}{A_{1}^{\prime}\left(0 ; c^{*}, \ell^{*} ; \gamma\right)}\left\{\frac{\beta_{I}^{\prime}\left(c^{*}\right)}{\left(V^{(2)}\right)_{z}\left(-\ell^{*}\right)}-\int_{-\ell^{*}}^{0} e^{-c^{*} z} A_{1}\left(z ; c^{*}, \ell^{*} ; \gamma\right)\left(V^{(2)}\right)_{z}(z) d z+\frac{\partial \ell}{\partial c}\right\} \\
& \quad+\frac{1}{A_{2}^{\prime}\left(0 ; c^{*}, m^{*} ; \gamma\right)}\left\{-\frac{\beta_{I}^{\prime}\left(-c^{*}\right)}{\left(V^{(3)}\right)_{z}\left(m^{*}\right)}\right. \\
& \left.\quad-\int_{0}^{m^{*}} e^{-c^{*} z} A_{2}\left(z ; c^{*}, m^{*} ; \gamma\right)\left(V^{(3)}\right)_{z}(z) d z-\frac{\partial m}{\partial c}\right\} \\
& =-\frac{V_{z}^{(2)}\left(-\ell^{*}\right)}{V_{z z}^{(2)}(0) e^{-c^{*} \ell^{*}}}\left[\beta_{I}^{\prime}\left(c^{*}\right)\left\{\frac{\left(V^{(1)}\right)_{z z}\left(-\ell^{*}\right)}{\left(V^{(1)}\right)_{z}\left(-\ell^{*}\right)}-\frac{\left(V^{(2)}\right)_{z z}\left(-\ell^{*}\right)}{\left(V^{(2)}\right)_{z}\left(-\ell^{*}\right)}\right\}\right. \\
& \left.\quad+\frac{\int_{-\infty}^{-\ell^{*}} e^{-c^{*}\left(z+\ell^{*}\right)}\left(\left(V^{(1)}\right)_{z}(z)\right)^{2} d z}{\left(V^{(1)}\right)_{z}\left(-\ell^{*}\right)}+\frac{\int_{-\ell^{*}}^{0} e^{-c^{*}\left(z+\ell^{*}\right)}\left(\left(V^{(2)}\right)_{z}(z)\right)^{2} d z}{\left(V^{(2)}\right)_{z}\left(-\ell^{*}\right)}\right] \\
& -\frac{V_{z}^{(3)}\left(-m^{*}\right)}{V_{z z}^{(3)}(0) e^{c^{*} m^{*}}}\left[-\beta_{I}^{\prime}\left(-c^{*}\right)\left\{\frac{\left(V^{(3)}\right)_{z z}\left(m^{*}\right)}{\left(V^{(3)}\right)_{z}\left(m^{*}\right)}-\frac{\left(V^{(4)}\right)_{z z}\left(m^{*}\right)}{\left(V^{(4)}\right)_{z}\left(m^{*}\right)}\right\}\right. \\
& \left.\quad+\frac{\int_{0}^{m^{*}} e^{-c^{*}\left(z-m^{*}\right)}\left(\left(V^{(3)}\right)_{z}(z)\right)^{2} d z}{\left(V^{(3)}\right)_{z}\left(m^{*}\right)}+\frac{\int_{m^{*}}^{\infty} e^{-c^{*}\left(z-m^{*}\right)}\left(\left(V^{(4)}\right)_{z}(z)\right)^{2} d z}{\left(V^{(4)}\right)_{z}\left(m^{*}\right)}\right]
\end{aligned}
$$




$$
\begin{aligned}
= & \frac{G\left[\beta_{1}\right]}{V_{z z}^{(2)}(0) e^{-c^{*} \ell^{*}}} \\
& \left\{-\frac{\int_{-\infty}^{-\ell^{*}} e^{-c^{*}\left(z+\ell^{*}\right)}\left(\left(V^{(1)}\right)_{z}(z)\right)^{2} d z+\int_{-\ell^{*}}^{0} e^{-c^{*}\left(z+\ell^{*}\right)}\left(\left(V^{(2)}\right)_{z}(z)\right)^{2} d z}{G\left[\beta_{1}\right]}-\beta_{I}^{\prime}\left(c^{*}\right)\right\} \\
+ & \frac{G\left[\beta_{2}\right]}{V_{z z}^{(3)}(0) e^{c^{*} m^{*}}} \\
& \left\{-\beta_{I}^{\prime}\left(-c^{*}\right)-\frac{\int_{0}^{m^{*}} e^{-c^{*}\left(z-m^{*}\right)}\left(\left(V^{(3)}\right)_{z}(z)\right)^{2} d z+\int_{m^{*}}^{\infty} e^{-c^{*}\left(z-m^{*}\right)}\left(\left(V^{(4)}\right)_{z}(z)\right)^{2} d z}{G\left[\beta_{2}\right]}\right\},
\end{aligned}
$$

where $G\left[\beta_{i}\right]=\left\{g\left(h_{+}\left(\beta_{i}\right), \beta_{i} ; \gamma\right)-g\left(h_{-}\left(\beta_{i}\right), \beta_{i} ; \gamma\right)\right\}>0(i=1,2)$. Unfortunately, we cannot evaluate the values of $\frac{\partial}{\partial c} L\left(c^{*} ; \gamma\right)$ and $\frac{d}{d \gamma} c(\gamma)$ for arbitrary $\gamma$ for which traveling front and back waves coexist. But we can do that in a small neighborhood of $\gamma_{1}$ with the aid of the properties of traveling front and back waves. First of all, noting that $\ell^{*}$ and $m^{*}$ tend to infinity as $\gamma$ tends to $\gamma_{1}$, we have the following asymptotic properties:

$$
\begin{aligned}
\left(V^{(2)}\right)_{z z}\left(0 ; c^{*}, \ell^{*} ; \gamma\right) & =\left(V_{f}^{+}\right)_{z z}\left(\ell^{*} ; c^{*} ; \tau, \gamma\right)(1+o(1)) \\
& =O\left(\exp \left(\frac{c^{*}-\sqrt{c^{* 2}-4 J_{+}\left(v_{+}(\gamma)\right)}}{2} \ell^{*}\right)\right) \\
\left(V^{(3)}\right)_{z z}\left(0 ; c^{*}, m^{*} ; \gamma\right) & =\left(V_{b}^{-}\right)_{z z}\left(-m^{*} ; c^{*} ; \tau, \gamma\right)(1+o(1)) \\
& =O\left(\exp \left(\frac{c^{*}+\sqrt{c^{* 2}-4 J_{+}\left(v_{+}(\gamma)\right)}}{2}\left(-m^{*}\right)\right)\right)
\end{aligned}
$$

as $\gamma \longrightarrow \gamma_{1}$, and

$$
\begin{aligned}
& V^{(1)}\left(z-\ell^{*} ; c^{*}, \ell^{*} ; \gamma\right)=V_{f}^{-}\left(z ; c^{*} ; \tau, \gamma\right)(1+o(1)) \quad \text { uniformly in }(-\infty, 0] \text {, } \\
& \left(V^{(1)}\right)_{z}\left(z-\ell^{*} ; c^{*}, \ell^{*} ; \gamma\right)=\left(V_{f}^{-}\right)_{z}\left(z ; c^{*} ; \tau, \gamma\right)(1+o(1)) \text { uniformly in }(-\infty, 0] \text {, } \\
& V^{(2)}\left(z-\ell^{*} ; c^{*}, \ell^{*} ; \gamma\right)=V_{f}^{+}\left(z ; c^{*} ; \tau, \gamma\right)(1+o(1)) \\
& \text { uniformly on any compact set in }[0, \infty) \text {, } \\
& \left(V^{(2)}\right)_{z}\left(z-\ell^{*} ; c^{*}, \ell^{*} ; \gamma\right)=\left(V_{f}^{+}\right)_{z}\left(z ; c^{*} ; \tau, \gamma\right)(1+o(1)) \\
& \text { uniformly on any compact set in }[0, \infty) \text {, } \\
& V^{(3)}\left(z+m^{*} ; c^{*}, m^{*} ; \gamma\right)=V_{b}^{-}\left(z ; c^{*} ; \tau, \gamma\right)(1+o(1)) \\
& \text { uniformly on any compact set in }(-\infty, 0] \text {, } \\
& \left(V^{(3)}\right)_{z}\left(z+m^{*} ; c^{*}, m^{*} ; \gamma\right)=\left(V_{b}^{-}\right)_{z}\left(z ; c^{*} ; \tau, \gamma\right)(1+o(1)) \\
& \text { uniformly any compact set in }(-\infty, 0] \text {, } \\
& V^{(4)}\left(z+m^{*} ; c^{*}, m^{*} ; \gamma\right)=V_{b}^{+}\left(z ; c^{*} ; \tau, \gamma\right)(1+o(1)) \text { uniformly in }[0, \infty) \text {, } \\
& \left(V^{(4)}\right)_{z}\left(z+m^{*} ; c^{*}, m^{*} ; \gamma\right)=\left(V_{b}^{+}\right)_{z}\left(z ; c^{*} ; \tau, \gamma\right)(1+o(1)) \text { uniformly in }[0, \infty) \text {, }
\end{aligned}
$$

as $\gamma \longrightarrow \gamma_{1}$, where $V_{f}^{ \pm}(z ; c ; \tau, \gamma)$ and $V_{b}^{ \pm}(z ; c ; \tau, \gamma)$ are the functions defined in $\S 2.4$ and $J_{+}(v)=g_{u}\left(h_{+}(v), v ; \gamma\right) h_{+}^{\prime}(v)+g_{v}\left(h_{+}(v), v ; \gamma\right)<0$. Then, when $\gamma$ tends to $\gamma_{1}$, using Remark 2.1, we can evaluate the above functions as follows: 


$$
\begin{aligned}
\frac{\partial}{\partial c} L\left(c^{*} ; \gamma\right)= & \frac{\partial Q}{\partial c}+\frac{\partial \ell}{\partial c} \cdot \frac{\partial Q}{\partial \ell}+\left.\frac{\partial m}{\partial c} \cdot \frac{\partial Q}{\partial m}\right|_{c=c^{*}, \ell=\ell^{*}, m=m^{*}} \\
= & {\left[\frac{G\left[\beta_{1}\right]}{\left(V_{f}^{+}\right)_{z z}\left(\ell^{*}\right) e^{-c^{*} \ell^{*}}}\left\{\beta_{O}^{\prime}\left(c^{*} ; \gamma\right)-\beta_{I}^{\prime}\left(c^{*}\right)\right\}\right.} \\
& \left.+\frac{G\left[\beta_{2}\right]}{\left(V_{b}^{-}\right)_{z z}\left(-m^{*}\right) e^{c^{*} m^{*}}}\left\{\beta_{O}^{\prime}\left(-c^{*} ; \gamma\right)-\beta_{I}^{\prime}\left(-c^{*}\right)\right\}\right](1+o(1)) .
\end{aligned}
$$

Here we should note that the first part in (4.24) comes from the transversality condition of the front wave and the second comes from that of the back wave. Furthermore, noting that

$$
\begin{aligned}
\left(V_{f}^{+}\right)_{z z}\left(\ell^{*}\right) e^{-c^{*} \ell^{*}} & =O\left(\exp \left(\frac{-c^{*}-\sqrt{c^{* 2}-4 J_{+}\left(v_{+}(\gamma)\right)}}{2} \ell^{*}\right)\right)(<0), \\
\left(V_{b}^{-}\right)_{z z}\left(-m^{*}\right) e^{c^{*} m^{*}} & =O\left(\exp \left(\frac{c^{*}-\sqrt{c^{* 2}-4 J_{+}\left(v_{+}(\gamma)\right)}}{2} m^{*}\right)\right)(<0),
\end{aligned}
$$

we find that the first (second) part in (4.24) is dominant when $c^{*}>0\left(c^{*}<0\right)$. Thus, for $\gamma$ in a small neighborhood of $P_{1}, P_{3}$ or $P_{5}$,

$$
\frac{\partial}{\partial c} L\left(c^{*} ; \gamma\right)=\frac{\partial Q}{\partial c}+\frac{\partial \ell}{\partial c} \cdot \frac{\partial Q}{\partial \ell}+\left.\frac{\partial m}{\partial c} \cdot \frac{\partial Q}{\partial m}\right|_{c=c^{*}, \ell=\ell^{*}, m=m^{*}}<0
$$

and for $\gamma$ in a small neighborhood of $P_{2}$,

$$
\frac{\partial}{\partial c} L\left(c^{*} ; \gamma\right)=\frac{\partial Q}{\partial c}+\frac{\partial \ell}{\partial c} \cdot \frac{\partial Q}{\partial \ell}+\left.\frac{\partial m}{\partial c} \cdot \frac{\partial Q}{\partial m}\right|_{c=c^{*}, \ell=\ell^{*}, m=m^{*}}>0
$$

Thus we have the following theorem:

Theorem 4.1. Bifurcated pulse waves at $P_{1}, P_{3}$, and $P_{5}$ have the crossing behavior with $\frac{\partial}{\partial c} L\left(c^{*}\right)<0$ and ones at $P_{2}$ have the crossing behavior with $\frac{\partial}{\partial c} L\left(c^{*}\right)>0$. (See Figure 11.)

Next, let us consider the following quantity:

$$
\begin{aligned}
& \frac{\partial Q}{\partial \gamma}+\frac{\partial \ell}{\partial \gamma} \cdot \frac{\partial Q}{\partial \ell}+\left.\frac{\partial m}{\partial \gamma} \cdot \frac{\partial Q}{\partial m}\right|_{c=c^{*}, \ell=\ell^{*}, m=m^{*}} \\
& =\frac{1}{A_{1}^{\prime}\left(0 ; c^{*}, \ell^{*} ; \gamma\right)}\left\{-\int_{-\ell^{*}}^{0} e^{-c^{*} z} A_{1}\left(z ; c^{*}, \ell^{*} ; \gamma\right)\left(V^{(2)}\right)_{z}(z) d z+\frac{\partial \ell}{\partial \gamma}\right\} \\
& \quad+\frac{1}{A_{2}^{\prime}\left(0 ; c^{*}, m^{*} ; \gamma\right)}\left\{-\int_{0}^{m^{*}} e^{-c^{*} z} A_{2}\left(z ; c^{*}, m^{*} ; \gamma\right)\left(V^{(3)}\right)_{z}(z) d z-\frac{\partial m}{\partial \gamma}\right\} \\
& =-\frac{1}{\left(V^{(2)}\right)_{z z}(0) e^{-c^{*} \ell^{*}}}\left\{\int_{-\infty}^{-\ell^{*}} e^{-c^{*}\left(z+\ell^{*}\right)}\left(V^{(1)}\right)_{z}(z) V^{(1)}(z) d z\right. \\
& \left.\quad+\int_{-\ell^{*}}^{0} e^{-c^{*}\left(z+\ell^{*}\right)}\left(V^{(2)}\right)_{z}(z) V^{(2)}(z) d z\right\} \\
& -\frac{1}{\left(V^{(3)}\right)_{z z}(0) e^{c^{*} m^{*}}}\left\{\int_{0}^{m^{*}} e^{-c^{*}\left(z-m^{*}\right)}\left(V^{(3)}\right)_{z}(z) V^{(3)}(z) d z\right. \\
& \left.\quad+\int_{m^{*}}^{\infty} e^{-c^{*}\left(z-m^{*}\right)}\left(V^{(4)}\right)_{z}(z) V^{(4)}(z) d z\right\}
\end{aligned}
$$




$$
\begin{aligned}
=-\left[\frac { 1 } { ( V _ { f } ^ { + } ) _ { z z } ( \ell ^ { * } ) e ^ { - c ^ { * } \ell ^ { * } } } \left\{\int_{-\infty}^{0} e^{-c^{*} z}\left(V_{f}^{-}\right)_{z}(z) V_{f}^{-}(z) d z\right.\right. \\
\left.\quad+\int_{0}^{\infty} e^{-c^{*} z}\left(V_{f}^{+}\right)_{z}(z) V_{f}^{+}(z) d z\right\} \\
+\frac{1}{\left(V_{b}^{-}\right)_{z z}\left(-m^{*}\right) e^{c^{*} m^{*}}}\left\{\int_{-\infty}^{0} e^{-c^{*} z}\left(V_{b}^{-}\right)_{z}(z) V_{b}^{-}(z) d z\right. \\
\left.\left.\quad+\int_{0}^{\infty} e^{-c^{*} z}\left(V_{b}^{+}\right)_{z}(z) V_{b}^{+}(z) d z\right\}\right] \times(1+o(1)) \quad \text { as } \gamma \rightarrow \gamma_{1} .
\end{aligned}
$$

If $c^{*}>0\left(c^{*}<0\right)$, the first (second) term of equations (4.24) and (4.27) is dominant. Then

$$
\begin{aligned}
& \left.\frac{d}{d \gamma} c_{p}^{+}(\gamma)\right|_{\gamma=\gamma_{1}}= \\
& \quad \frac{\left\{\int_{-\infty}^{0} e^{-c_{p}^{+} z}\left(V_{f}^{-}\right)_{z}(z) V_{f}^{-}(z) d z+\int_{0}^{\infty} e^{-c_{p}^{+} z}\left(V_{f}^{+}\right)_{z}(z) V_{f}^{+}(z) d z\right\}}{G\left[\beta_{1}\right]\left\{\beta_{O}^{\prime}\left(c_{p}^{+} ; \gamma_{1}\right)-\beta_{I}^{\prime}\left(c_{p}^{+}\right)\right\}}(1+o(1))
\end{aligned}
$$

and

$$
\begin{aligned}
& \left.\frac{d}{d \gamma} c_{p}^{-}(\gamma)\right|_{\gamma=\gamma_{1}}= \\
& \quad \frac{\left\{\int_{-\infty}^{0} e^{-c_{p}^{-} z}\left(V_{b}^{-}\right)_{z}(z) V_{b}^{-}(z) d z+\int_{0}^{\infty} e^{-c_{p}^{-} z}\left(V_{b}^{+}\right)_{z}(z) V_{b}^{+}(z) d z\right\}}{G\left[\beta_{2}\right]\left\{\beta_{O}^{\prime}\left(-c_{p}^{-} ; \gamma_{1}\right)-\beta_{I}^{\prime}\left(-c_{p}^{-}\right)\right\}}(1+o(1)) .
\end{aligned}
$$

On the other hand, note that the curve $c=c_{f}(\gamma)$ was determined by the relation

$$
\beta_{I}(c)=\beta_{O}(c ; \gamma) \text {. }
$$

Then, substituting $c=c_{f}(\gamma)$ into this relation and differentiating with respect to $\gamma$, we have

$$
\frac{\partial}{\partial \gamma} c_{f}(\gamma)=\left.\frac{\frac{\partial}{\partial \gamma} \beta_{O}(c ; \gamma)}{\beta_{I}^{\prime}(c)-\beta_{O}^{\prime}(c ; \gamma)}\right|_{c=c_{f}(\gamma)}
$$

Remark 2.1 implies that

$$
\left.\frac{d}{d \gamma} c_{p}^{+}(\gamma)\right|_{\gamma=\gamma_{1}}=\left.\frac{\partial}{\partial \gamma} c_{f}(\gamma)\right|_{\gamma=\gamma_{1}}
$$

Similarly, we have

$$
\left.\frac{d}{d \gamma} c_{p}^{-}(\gamma)\right|_{\gamma=\gamma_{1}}=\left.\frac{\partial}{\partial \gamma} c_{b}(\gamma)\right|_{\gamma=\gamma_{1}}
$$

If $c^{*}=0$ in (4.27),

$$
\frac{\partial Q}{\partial \gamma}+\frac{\partial \ell}{\partial \gamma} \cdot \frac{\partial Q}{\partial \ell}+\left.\frac{\partial m}{\partial \gamma} \cdot \frac{\partial Q}{\partial m}\right|_{c=c^{*}, \ell=\ell^{*}, m=m^{*}, \gamma=\gamma_{1}}=0
$$

Therefore, we get

$$
\left.\frac{d}{d \gamma} c_{p}^{0}(\gamma)\right|_{\gamma=\gamma_{1}}=0
$$




\section{Concluding remarks}

In this paper, by analyzing the singular limit problem of the reaction-diffusion system, we proved Theorem 3.4 (see Figure 11). That is, in a neighborhood of $P_{i}(i=1,2,3,5)$, $P$-pulse waves and $Q$-pulse waves bifurcate at $\gamma=\gamma_{1}$. A more detailed proof of the existence of an exact solution will be given in [4]. There, the transversality condition will play the essential role. Also in [4], stability properties of these traveling pulse waves are discussed with the aid of Theorem 4.1. Applying singular perturbation methods to the linearized eigenvalue problem leads to the algebraic equation $G(\lambda ; \gamma)=$ 0 relating the eigenvalues $\lambda$ and the parameter $\gamma$. Finding roots $\lambda$ satisfying $G(\lambda ; \gamma)=$ 0 is equivalent to solving the linearized eigenvalue problem. When $\gamma=\gamma_{1}$, a traveling pulse wave is separated into a traveling front wave and a traveling back one. Then, the distribution of roots satisfying $G\left(\lambda ; \gamma_{1}\right)=0$ depends on the stabilities of the front and back waves. That is, for example, if the front and back waves are both stable (unstable), $G\left(\lambda ; \gamma_{1}\right)=0$ has double zero roots and double negative (positive) roots $\lambda^{*}$ (see Figure 12(a-i), (b-i)). When $\gamma$ is slightly perturbed from $\gamma=\gamma_{1}$, how does the distribution of the roots satisfying $G\left(\lambda ; \gamma_{1}\right)=0$ change?

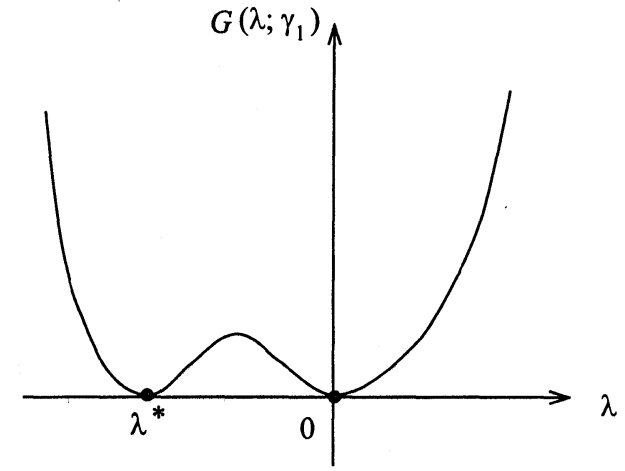

(a-i)

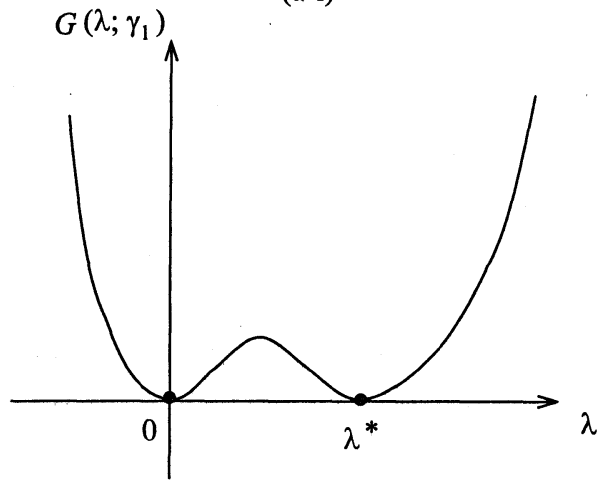

(b-i)

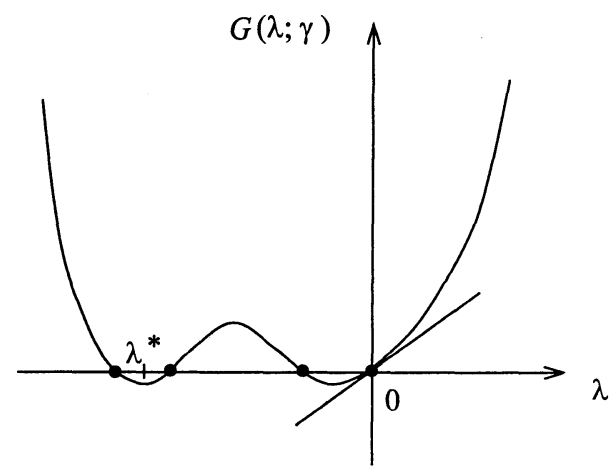

(a-ii)

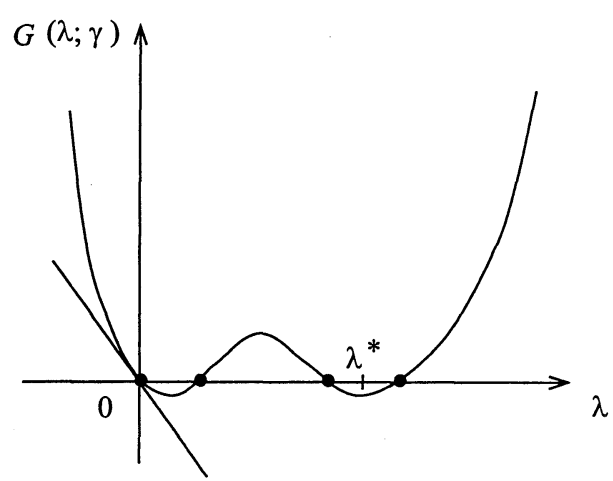

(b-ii)

FIgURE 12. Distribution of eigenvalues: (a) stable front and stable back waves: (i) $\gamma=\gamma_{1}$, (ii) $\gamma \sim \gamma_{1}$, (b) unstable front and unstable back waves: (i) $\gamma=\gamma_{1}$, (ii) $\gamma \sim \gamma_{1}$.

Two nonzero roots still stay in a neighborhood of $\lambda^{*}$. Moreover, due to translation invariance of a traveling pulse wave, we find that $G(\lambda ; \gamma)=0$ has one zero root which 
remains after the perturbation of $\gamma$ from $\gamma_{1}$. Therefore, the most important problem is to determine the sign of the eigenvalue which is perturbed from the other zero root for the case $\gamma=\gamma_{1}$. For this problem, we have the relation

$$
\operatorname{sgn}\left\{\frac{\partial}{\partial \lambda} G(0 ; \gamma)\right\}=\operatorname{sgn}\left\{-\frac{\partial}{\partial c} L\left(c^{*} ; \gamma\right)\right\},
$$

where $\frac{\partial}{\partial c} L\left(c^{*} ; \gamma\right)$ is the crossing behavior given in Theorem 4.1 (see Figure 12). Thus, we can show that one traveling pulse wave which bifurcates from the intersecting point of a stable front wave and a stable back one is stable, and other types of traveling pulse waves are unstable. That is, traveling pulse waves which bifurcate at $P_{1}, P_{3}$, and $P_{5}$ are stable, and ones at $P_{2}$ are unstable. Generally speaking, the stability of a traveling pulse wave is determined by the following two items: the first is the stability of front and back waves, which become parts of the pulse wave. The other is how to connect two parts, which comes from the crossing behavior of the stable and unstable manifolds.

Let us revise Figure 11 to give the diagrams of the relation $c$ versus $\tau$. Then we obtain Figure 13.

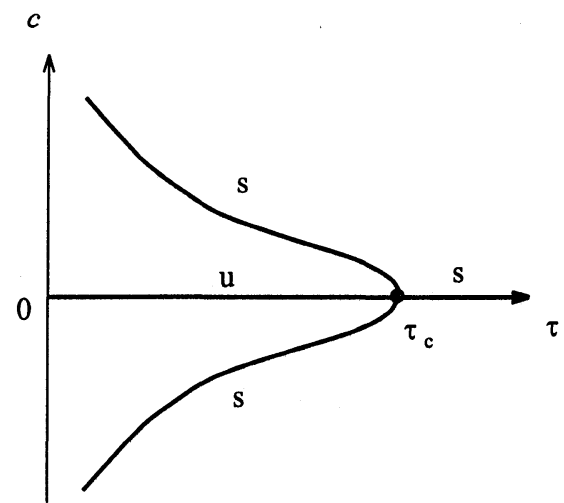

(a)

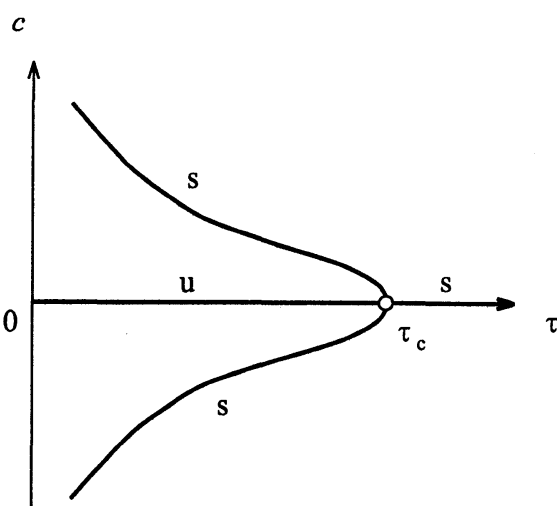

(b)

FIGURE 13. Singular limit velocity curves $c$ versus $\tau$ : (a) traveling front and back waves at $\gamma=\gamma_{1}$, (b) traveling pulse waves at $\gamma \sim \gamma_{1}$.

The label "s" means stable and " $u$ " means unstable. Finally, we note that the dimension of the unstable manifold of the unstable standing pulse wave is equal to three. Figure 13(b) suggests that traveling pulse waves bifurcate from standing pulse waves at $\tau=\tau_{c}$. But numerical simulations indicate a more complex situation in the neighborhood of $\tau=\tau_{c}$. This is a problem to be considered in the future.

\section{Appendix}

Proof of Lemma 4.1. We only show the relations for the solutions $V^{(1)}(z ; c, \ell ; \gamma)$ and $V^{(2)}(z ; c, \ell ; \gamma)$ since the other cases can be obtained similarly. Using the variable $y=z+\ell$ and putting $w(y ; c ; \gamma)=V^{(1)}(y-\ell ; c, \ell ; \gamma)$, (4.1a) may be rewritten as

$$
\begin{aligned}
& w_{y y}-c w_{y}+h_{-}(w)-\gamma w=0 \quad y \in(-\infty, 0) \equiv \mathbf{R}_{-}, \\
& w(-\infty)=v_{-}(\gamma), \quad w(0)=\beta_{I}(c),
\end{aligned}
$$


where we used the relationship $g(u, v ; \gamma)=u-\gamma v$. Differentiating (A.1) with respect to $c$, we find that $p=\frac{\partial w}{\partial c}$ satisfies

$$
\begin{aligned}
& p_{y y}-c p_{y}+\left\{h_{-}^{\prime}(w)-\gamma\right\} p=w_{y} \quad y \in \mathbf{R}_{-}, \\
& p(-\infty)=0, \quad p(0)=\beta_{I}^{\prime}(c) .
\end{aligned}
$$

Then $p$ is explicitly represented as

$$
p(y ; c ; \gamma)=\frac{\beta_{I}^{\prime}(c) w_{y}(y)}{w_{y}(0)}-w_{y}(y) \int_{y}^{0} e^{c x}\left(w_{x}(x)\right)^{-2} \int_{-\infty}^{x} e^{-c t}\left(w_{t}(t)\right)^{2} d t d x .
$$

Therefore, differentiating by $y$ and replacing $w$ by $V^{(1)}$, we find that

$$
\begin{aligned}
\frac{\partial}{\partial c}\left(V^{(1)}\right)_{z}(-\ell ; c, \ell ; \gamma)= & \beta_{I}^{\prime}(c)\left(V^{(1)}\right)_{z z}(-\ell) /\left(V^{(1)}\right)_{z}(-\ell) \\
& +\int_{-\infty}^{-\ell} e^{-c(z+\ell)}\left(\left(V^{(1)}\right)_{z}(z)\right)^{2} d z /\left(V^{(1)}\right)_{z}(-\ell) .
\end{aligned}
$$

Similar to the above, we have

$$
\frac{\partial}{\partial \gamma}\left(V^{(1)}\right)_{z}(-\ell ; c, \ell ; \gamma)=\int_{-\infty}^{-\ell} e^{-c(z+\ell)}\left(V^{(1)}\right)_{z}(z) V^{(1)}(z) d z /\left(V^{(1)}\right)_{z}(-\ell)
$$

If we differentiate $w(y ; c ; \gamma)=V^{(1)}(y-\ell ; c, \ell ; \gamma)$ with respect to $\ell$ and put $y=0$, we have

$$
\frac{\partial}{\partial \ell}\left(V^{(1)}\right)_{z}(-\ell ; c, \ell ; \gamma)=\left(V^{(1)}\right)_{z z}(-\ell)
$$

Next, we consider the problem (4.1b). $p=\frac{\partial V^{(2)}}{\partial c}$ satisfies the equations

$$
\begin{aligned}
& p_{z z}-c p_{z}+\left\{h_{+}^{\prime}\left(V^{(2)}\right)-\gamma\right\} p=\left(V^{(2)}\right)_{z} \quad z \in(-\ell, 0), \\
& p(-\ell)=\beta_{I}^{\prime}(c), \quad p_{z}(0)=0 .
\end{aligned}
$$

Let us define two functions

$$
\begin{aligned}
& A_{1}(z ; c, \ell ; \gamma)=\left(V^{(2)}\right)_{z}(z ; c, \ell ; \gamma) \int_{-\ell}^{z} e^{c y}\left(\left(V^{(2)}\right)_{y}(y ; c, \ell ; \gamma)\right)^{-2} d y \\
& B_{1}(z ; c, \ell ; \gamma)=\left(V^{(2)}\right)_{z}(z ; c, \ell ; \gamma)-\frac{\left(V^{(2)}\right)_{z z}(0 ; c, \ell ; \gamma) A_{1}(z ; c, \ell ; \gamma)}{A_{1}^{\prime}(0 ; c, \ell ; \gamma)}
\end{aligned}
$$

where $A_{1}^{\prime}(0 ; c, \ell ; \gamma)=\frac{d}{d z} A_{1}(0 ; c, \ell ; \gamma)>0$. We easily see that

$$
G(z, y ; c, \ell ; \gamma)= \begin{cases}A_{1}(z ; c, \ell ; \gamma) B_{1}(y ; c, \ell ; \gamma), & -\ell \leq z<y \\ B_{1}(z ; c, \ell ; \gamma) A_{1}(y ; c, \ell ; \gamma), & y<z \leq 0\end{cases}
$$

is the Green's function for the problem

$$
\begin{gathered}
\left(e^{-c z} p_{z}\right)_{z}+e^{-c z}\left\{h_{+}^{\prime}\left(V^{(2)}\right)-\gamma\right\} p=0, \quad z \in(-\ell, 0), \\
p(-\ell)=0, \quad p_{z}(0)=0 .
\end{gathered}
$$

Using this Green's function, we can find the solution of (A.2) explicitly:

$$
p(z ; c, \ell ; \gamma)=\beta_{I}^{\prime}(c) \frac{B_{1}(z ; c, \ell ; \gamma)}{B_{1}(-\ell ; c, \ell ; \gamma)}-\int_{-\ell}^{0} G(z, y ; c, \ell ; \gamma) e^{-c y}\left(V^{(2)}\right)_{y}(y) d y
$$


From this formula, the following two relations hold:

$$
\begin{aligned}
\frac{\partial}{\partial c} V^{(2)}(0 ; c, \ell ; \gamma)= & \frac{1}{A_{1}^{\prime}(0 ; c, \ell ; \gamma)}\left\{\frac{\beta_{I}^{\prime}(c)}{\left(V^{(2)}\right)_{z}(-\ell)}\right. \\
& \left.-\int_{-\ell}^{0} e^{-c z} A_{1}(z ; c, \ell ; \gamma)\left(V^{(2)}\right)_{z}(z) d z\right\} \\
\frac{\partial}{\partial c}\left(V^{(2)}\right)_{z}(-\ell ; c, \ell ; \gamma)= & \frac{\beta_{I}^{\prime}(c)}{\left(V^{(2)}\right)_{z}(-\ell)}\left\{\left(V^{(2)}\right)_{z z}(-\ell)-\frac{\left(V^{(2)}\right)_{z z}(0)}{A_{1}^{\prime}(0 ; c, \ell ; \gamma)} \cdot \frac{e^{-c \ell}}{\left(V^{(2)}\right)_{z}(-\ell)}\right\} \\
& -\frac{e^{-c \ell}}{\left(V^{(2)}\right)_{z}(-\ell)}\left\{\int_{-\ell}^{0} e^{-c z}\left(\left(V^{(2)}\right)_{z}(z)\right)^{2} d z\right. \\
& \left.-\frac{\left(V^{(2)}\right)_{z z}(0)}{A_{1}^{\prime}(0 ; c, \ell ; \gamma)} \int_{-\ell}^{0} e^{-c z} A_{1}(z ; c, \ell ; \gamma)\left(V^{(2)}\right)_{z}(z) d z\right\} .
\end{aligned}
$$

Similar to the above, we have

$$
\begin{aligned}
\frac{\partial}{\partial \gamma} V^{(2)}(0 ; c, \ell ; \gamma) & =-\frac{1}{A_{1}^{\prime}(0 ; c, \ell ; \gamma)} \int_{-\ell}^{0} e^{-c z} A_{1}(z ; c, \ell ; \gamma) V^{(2)}(z) d z \\
\frac{\partial}{\partial \gamma}\left(V^{(2)}\right)_{z}(-\ell ; c, \ell ; \gamma) & =-\frac{e^{-c \ell}}{\left(V^{(2)}\right)_{z}(-\ell)}\left\{\int_{-\ell}^{0} e^{-c z}\left(V^{(2)}\right)_{z}(z) V^{(2)}(z) d z\right. \\
& \left.+\frac{\left(V^{(2)}\right)_{z z}(0)}{A_{1}^{\prime}(0 ; c, \ell ; \gamma)} \int_{-\ell}^{0} e^{-c z} A_{1}(z ; c, \ell ; \gamma) V^{(2)}(z) d z\right\}
\end{aligned}
$$

Finally, we consider the dependence of $\ell$ on $V^{(2)}(z ; c, \ell ; \gamma)$. Because $\ell$ depends on the interval $(-\ell, 0)$, using the variable $y=z / \ell$, we rewrite $(4.1 \mathrm{~b})$ in the following form where $w(y ; c, \ell ; \gamma)=V^{(2)}(\ell y ; c, \ell ; \gamma)$ :

$$
\begin{aligned}
& w_{y y}-c \ell w_{y}+\ell^{2}\left\{h_{+}(w)-\gamma w\right\}=0 \quad y \in(-1,0) \\
& w(-1)=\beta_{I}(c), \quad w_{y}(0)=0 .
\end{aligned}
$$

Then $q=\frac{\partial w}{\partial \ell}$ satisfies

$$
\begin{aligned}
& q_{y y}-c \ell q_{y}+\ell^{2}\left\{h_{+}^{\prime}(w)-\gamma\right\} q=c w_{y}-2 \ell\left\{h_{+}(w)-\gamma w\right\} \quad y \in(-1,0) \\
& q(-1)=0, \quad q_{y}(0)=0 .
\end{aligned}
$$

Here we rewrite (A.3) using the original independent variable $z$ again. Differentiate the relation

$$
w(y ; c, \ell ; \gamma)=V^{(2)}(\ell y ; c, \ell ; \gamma)\left(=V^{(2)}(z ; c, \ell ; \gamma)\right)
$$

with respect to $\ell$. We have

$$
q(y ; c, \ell ; \gamma)=\left(V^{(2)}\right)_{z}(\ell y ; c, \ell ; \gamma) y+\frac{\partial V^{(2)}}{\partial \ell}(\ell y ; c, \ell ; \gamma)
$$

Furthermore, we make use of the relations

$$
\begin{aligned}
q_{y}(y ; c, \ell ; \gamma)= & \left(V^{(2)}\right)_{z z}(\ell y ; c, \ell ; \gamma) \ell y+\left(V^{(2)}\right)_{z}(\ell y ; c, \ell ; \gamma)+\frac{\partial}{\partial \ell}\left(V^{(2)}\right)_{z}(\ell y ; c, \ell ; \gamma) \ell \\
q_{y y}(y ; c, \ell ; \gamma)= & \left(V^{(2)}\right)_{z z z}(\ell y ; c, \ell ; \gamma) \ell^{2} y+2\left(V^{(2)}\right)_{z z}(\ell y ; c, \ell ; \gamma) \ell \\
& +\frac{\partial}{\partial \ell}\left(V^{(2)}\right)_{z z}(\ell y ; c, \ell ; \gamma) \ell^{2}
\end{aligned}
$$


Let $p=\frac{\partial V^{(2)}}{\partial \ell}$. (A.3) may be rewritten as

$$
\begin{array}{rr}
p_{z z}-c p_{z}+\left\{h_{+}^{\prime}\left(V^{(2)}\right)-\gamma\right\} p=0 & z \in(-\ell, 0) \\
p(-\ell)=\left(V^{(2)}\right)_{z}(-\ell), & p_{z}(0)=0
\end{array}
$$

Then, we see

$$
\frac{\partial V^{(2)}}{\partial \ell}(z ; c, \ell ; \gamma)=\frac{\left(V^{(2)}\right)_{z}(-\ell ; c, \ell ; \gamma) B_{1}(z ; c, \ell ; \gamma)}{B_{1}(-\ell ; c, \ell ; \gamma)}=B_{1}(z ; c, \ell ; \gamma)
$$

from which we have

$$
\begin{gathered}
\frac{\partial}{\partial \ell} V^{(2)}(0 ; c, \ell ; \gamma)=\frac{1}{A_{1}^{\prime}(0 ; c, \ell ; \gamma)} \\
\frac{\partial}{\partial \ell}\left(V^{(2)}\right)_{z}(-\ell ; c, \ell ; \gamma)=\left(V^{(2)}\right)_{z z}(-\ell)-\frac{\left(V^{(2)}\right)_{z z}(0)}{A_{1}^{\prime}(0 ; c, \ell ; \gamma)} \cdot \frac{e^{-c \ell}}{\left(V^{(2)}\right)_{z}(-\ell)}
\end{gathered}
$$

Thus the proof of Lemma 4.1 is completed.

Acknowledgments. This research was done in part at the Department of Mathematics, University of British Columbia, and supported by the Japan Society for the Promotion of Science and the Natural Sciences and Engineering Research Council of Canada. The author is grateful to Professor Masayasu Mimura for useful comments. Special thanks are given to Professor Robert Miura for stimulating discussions during my visit to the University of British Columbia.

\section{References}

1. J. D. Dockery and J. P. Keener, Diffusive effects on dispersion in excitable media, SIAM J. Appl. Math. 49 (1989), 539-566.

2. P. C. Fife and J. B. McLeod, The approach of solutions of nonlinear diffusion equation to travelling wave solutions, Arch. Rat. Mech. Anal. 65 (1977), 335-361.

3. J. Guckenheimer and P. Holmes, Nonlinear Oscillations, Dynamical Systems, and Bifurcations of Vector Fields, Appl. Math. Sci. 42, Springer-Verlag, New York, 1983.

4. H. Ikeda, Existence and stability of pulse waves bifurcated from front and back waves in bistable reaction-diffusion systems, preprint.

5. H. Ikeda, M. Mimura, and Y. Nishiura, Global bifurcation phenomena of traveling wave solutions for some bistable reaction-diffusion systems, Nonlinear Anal. TMA 13 (1989), 507-526.

6. H. Kokubu, Y. Nishiura, and H.Oka, Heteroclinic and homoclinic bifurcations in bistable reactiondiffusion systems, J. Diff. Eqs. 86 (1990), 260-341.

7. Y. Nishiura, M. Mimura, H. Ikeda, and H. Fujii, Singular limit analysis of stability of traveling wave solutions in bistable reaction-diffusion systems, SIAM J. Math. Anal. 21, (1990), 85-122.

Department of Mathematics, Toyama University, Toyama 930, Japan 